\title{
POTENTIAL THEORY ON TREES, GRAPHS AND AHLFORS-REGULAR METRIC SPACES
}

\author{
NICOLA ARCOZZI, RICHARD ROCHBERG, ERIC T. SAWYER, AND BRETT D. WICK
}

ABSTRACT. We investigate connections between potential theories on a Ahlfors-regular metric space $X$, on a graph $G$ associated with $X$, and on the tree $T$ obtained by removing the "horizontal edges" in $G$. Applications to the calculation of set capacity are given.

\section{Contents}

1. Introduction 2

1.1. Main Results and Outline of the Contents 4

1.2. Some Examples of Ahlfors-Regular Metric Spaces. 6

2. A Metric Space, a Graph, and a Tree 7

2.1. Christ's Theorem Revisited 7

2.2. The space $X$ as the Boundary of $G \quad 10$

2.3. The Tree and its Boundary as Metric Spaces 13

2.4. The Map $\Lambda: \partial T \rightarrow X \quad 14$

2.5. Moving Measures through $\Lambda \quad 15$

3. The Muckenhoupt-Wheeden Inequality on Graphs 20

4. Proof of Theorem 1

5. Potential Theory on Trees 26

5.1. Tree Capacity Seen from "Inside" the Tree. 27

5.2. Capacity of Special Sets. 29

5.3. An Algorithm to Compute Tree Capacities 30

5.4. Trace Inequalities on Trees and Ahlfors-regular spaces. 32

5.5. Defining Capacities via Carleson measures 36

5.6. Monotonicity of the Tree Condition 38

5.7. Trace Inequalities: The Testing Condition implies the Capacitary Condition 39

6. Appendix: Potential Theory on Trees 41

References 43

Date: September 6, 2018.

2010 Mathematics Subject Classification. XXXX.

Key words and phrases. Ahlfors-regular metric space, potential theory, capacity.

The first author's work partially supported by the COFIN project Analisi Armonica, funded by the Italian Minister for Research.

The second author's work supported by the National Science Foundation under Grant No. 0700238.

The third author's work supported by the National Science and Engineering Council of Canada.

The fourth author's work supported by the National Science Foundation under Grant No. 1001098 and 0955432. 


\section{INTRODUCTION}

One of the cornerstones of potential theory is the notion of set capacity, which plays there a rôle analogous to that played by Lebesgue measure, or Haar measure, in the theory of $L^{p}$ spaces and harmonic analysis, to oversimplify a bit. The notion of capacity has its origins in physics, where it measures the maximum amount of (positive, say) electric charge which can be carried by a conductor while keeping the potential generated by that charge below a fixed threshold. The notion of capacity has been extended to nonlinear potentials, to various metric space settings, to the theory of stochastic processes and more. Since a long time, capacity had its peculiar rôle in the theory of conformal mappings and it is foundational material for the theory of quasi-conformal mappings. There is an extensive literature on these topics and we merely refer the reader to a tiny sample of it [Ts] [AH] [Mazya] [Doob] [He].

A basic fact about set capacity is that it is subadditive, but not additive; to the point that there are sets of positive, finite capacity with different Hausdorff dimensions. Capacity, that is, is much different from, although linked with, Hausdorff measure. This fact makes it computing, or just estimating, set capacity a craft on its own. The capacity of a set, in fact, measures together its geometric size and the way its pieces are distributed in the surrounding space. The bed of nails of a fakir is a well known example: cleverly arranged nails sum up to little area, but they might be distributed so as to have sufficient capacity not to break the membrane which is laid on them.

There is one context, however, where an elementary algorithm to compute capacity exists, and that is the context of trees. The capacity of subsets of the tree's boundary, in fact, reduces to the calculation of continued fractions of generalized type. This fact seems to have been independently observed by all researchers who, for different reasons, developed some potential theory on trees [LyPe][So][ARS3]. It is interesting then to know if these simple calculations on trees can be of some help in estimating capacities of sets in, say, Euclidean space.

In this article we show that this is in fact possible in the rather general context of Ahlfors regular metric spaces, for $p$-capacities associated with suitable Bessel-type kernels. To each such space $X$ we associate a tree $T$ : the capacity of a closed subset of $X$ turns out to be estimated from above and below by the capacity of a corresponding closed subset of $T$ 's boundary. The result seems new even for Bessel capacity in Euclidean space $\mathbb{R}^{n}, n \geq 2$. For linear capacities and $n=1$ it was proved by Benjamini and Peres in [BP]. For $n=1$ and $p \neq 2$ it follows rather directly from the results in [VW]. The boundary $\partial T$ of the tree $T$ is a totally disconnected set with respect to a natural metric. The problem of estimating set capacities is reduced then to an analogous problem for subsets of a generalized Cantor set.

The interest of the result, we believe, goes beyond the problem of estimating set capacities per se. Many problems in potential theory have an essentially combinatorial nature, which is best seen when they are translated in the language of trees.

Our interest in such questions developed while the first three authors were working with Carleson measures for Dirichlet-type spaces of holomorphic functions on the unit disc. They can can be characterized by means of a condition involving logarithmic capacity [Ste] or by a discrete testing-type condition making use of the tree structure of the disc's Whitney decomposition [ARS1]. A direct proof that testing conditions and capacitary conditions are equivalent in the tree context is in [ARS3]. This is evidence that a strict interplay exists in potential theory between some metric spaces and the trees obtained by discretizing them. 
Observations of a similar flavor have been made before. In [BP], Benjamini and Peres proved that the recurrence/transience dichotomy for some random walks on trees can be decided in terms of logarithmic capacity in the Euclidean plane where the trees have been suitably imbedded. In [VW], Verbitsky and Wheeden proved in some generality that the study of nonlinear potentials can be reduced to "dyadic" potentials. In retrospect, it might be said that the equivalence between dyadic and continuous potentials was implicit in Wolff's proofs in $[\mathrm{HW}]$, and this article might be seen as a long commentary on Wolff's work.

The linear, one-dimensional, Euclidean version of the results that are here discussed in greater generality is used in [ARSW] to prove a Nehari-type theorem for bilinear forms on the holomorphic Dirichlet space. In $[\mathrm{A}]$ it is used to find asymptotic estimates of some condenser capacities in the complex plane. The latter can be extended to higher dimensions using the results of the present article $[\mathrm{AB}]$.

There are three main tools which we use to build the bridge between trees and Ahlforsregular spaces. The first is Christ's dyadic decomposition of a homogeneous metric space [Christ]; or, rather, the easy part of it. We refine it, in the more specialized Ahlfors-regular case, to make room for the singular measures which are necessary in potential theory. Christ's construction can be thought of as a graph $G$ representing the geometry of $X$ at different scales. In particular, we identify $X$ with the bi-Lipschitz image of the graph's boundary, a result which might have independent interest. The tree $T$ used by Christ in his study of singular integrals is a spanning tree for the graph $G$. A construction closely related to ours was carried out in $[\mathrm{BoPa}] \S 2$. We thank the anonymous referee for directing our attention on this and other references.

The second tool, which is nedded if $X$ is not homeomorphic to a subset of the real line, is a technical lemma showing that measures can be moved back and forth from the metric space $X$ to the boundary of its associated tree. We must define the (non-canonical) pullback of a measure and this poses a nontrivial measurability problem. The third tool, useful to deal with the nonlinear case, is a deep inequality by Muckenhoupt and Wheeden [MW], later independently proved by Wolff $[\mathrm{HW}]$ with a wholly different, almost combinatorial argument. The Muckenhoupt-Wheeden-Wolff inequality, in fact, moves potentials from the space $X$ to the graph $G$, where it is easy to see that the "vertical edges", those which define the tree structure, carry all the relevant quantitative information. The inequality of Muckenhoupt, Wheeden and Wolff extends the well known equivalence, for subsets of the real line, of planar logarithmic capacity and one-dimensional 1/2-Bessel capacity. In the general case, this equivalence can be stated in terms of Wolff potentials.

In order to better focus on the main ideas, in this article we only consider the case of bounded, complete Ahlfors-regular spaces. The theory could be extended to the unbounded case by choosing Bessel-type potentials with exponential decay at infinity, in order to control the tail estimates. A good source for this kind of extension is [KV].

We finish with a comment about the bibliography. We have made references to articles and books where the results we needed or we made reference to could be found. We did not look for the primary source of the results we have quoted and we have probably omitted some important references. This is especially true for the section concerning potential theory on trees. Many of the results which we prove in Section 5 can be found in the literature, often independently proved by several authors at different times, including the authors of the present paper, with different degrees of generality; or they might be seen as particular cases of general results in one of the several axiomatic versions of Potential Theory. We have 
chosen to prove ourselves what we needed, trying to keep the exposition as self-contained as possible. There are two exceptions: the proof of Christ's Decomposition Theorem, for which we refer to [Christ], and the basic properties of Axiomatic Potential Theory, which we took from $[\mathrm{AH}]$. The main point of the paper, in fact, is not developing some new Potential Theory on Trees, but rather shedding light on the connection between discrete and nondiscrete Potential Theory. Some of our results on trees might nonetheless be new, especially those relating capacity and Carleson measures.

We thank the careful referee for pointing out a number of typos in the original draft of the paper and for his/her numerous suggestions on how to improve the presentation.

1.1. Main Results and Outline of the Contents. Let $(X, m, \rho)$ be a Ahlfors-regular metric measure space. By this we mean that $(X, \rho)$ is a complete metric space, $m \geq 0$ is a Borel measure on $X$, and there exist constants $0<c_{1}<c_{2}, Q>0$, such that, for all $r \geq 0$ and $x \in X$ :

$$
c_{1} r^{Q} \leq m(B(x, r)) \leq c_{2} r^{Q} .
$$

Here, $B(x, r)=\{y \in X: \rho(x, y)<\rho\}$ is the metric ball of radius $r$, centered at $x$.

In this article we are interested in local properties and we frequently will assume that $\operatorname{diam}(X)<+\infty$. In this case, (1) is only required to hold for $0 \leq r \leq \operatorname{diam}(X)$. This special assumption could be removed by assuming that the potential kernel $K$ below has exponential decay, analogous to that of the Bessel kernels in $\mathbb{R}^{n}$.

Given $0<s<1$, define the kernel $K: X \times X \rightarrow[0, \infty]$,

$$
K(x, y)=[m(B(x, \rho(x, y)))+m(B(y, \rho(x, y)))]^{-s} \approx m(x, \rho(x, y))^{-s} .
$$

When $X$ is an Ahlfors-regular, bounded domain in $\mathbb{R}^{n}$, the kernel $K$ is a Riesz-Bessel kernel:

$$
K(x, y)=\frac{1}{|x-y|^{s n}} .
$$

Given $1<p, p^{\prime}<\infty, \frac{1}{p}+\frac{1}{p^{\prime}}=1$, and a Borel measure $\omega \geq 0$ on $X$, consider the $p$-energy of $\omega$ associated with the kernel $K$ :

$$
\mathcal{E}_{X}(\omega):=\int_{X}[K \omega(x)]^{p^{\prime}} d m(x),
$$

where $K \omega(x)=\int_{X} K(x, y) d \omega(y)$. The $p$-capacity relative to the kernel $K$ associates to $E$, a compact subset of $X$, the nonnegative number

$$
\operatorname{Cap}_{X}(E)=\sup _{\operatorname{supp}(\omega) \subseteq E}\left[\frac{\omega(E)^{p}}{\mathcal{E}_{X}(\omega)^{p-1}}\right] .
$$

See $[\mathrm{AH}][\mathrm{He}][\mathrm{HaK}][\mathrm{HeK}][\mathrm{KV}]$ for various approaches to nonlinear capacities in measure metric spaces. The kind of potential theory which is discussed in this paper is only interesting when the parameter of smoothness $s$ satisfies $\frac{1}{p^{\prime}} \leq s<1$. When $0<s<\frac{1}{p^{\prime}}$, singletons have positive capacity.

We will show that capacities in Ahlfors-regular metric spaces can be estimated by similar capacities defined on a totally disconnected metric space. 
Theorem 1. To $(X, m, \rho)$ we can associate a tree $T$, a metric $\rho_{T}$ on $\partial T$ with respect to which $\partial T$, the boundary of $T$, is Ahlfors $Q$-regular, and a Lipschitz map

$$
\Lambda: \partial T \rightarrow X
$$

in such a way that, if $E$ is compact in $X$, then

$$
\operatorname{Cap}_{X}(E) \approx \operatorname{Cap}_{\partial T}\left(\Lambda^{-1}(E)\right) .
$$

In the other direction we have that, if $F$ is compact in $\partial T$, then

$$
\operatorname{Cap}_{X}(\Lambda(F)) \approx \operatorname{Cap}_{\partial T}(F)
$$

Here, $\operatorname{Cap}_{\partial T}$ is the capacity on the measure metric space $\left(\partial T, m_{T}, \rho_{T}\right)$, defined by the same parameters used in defining the capacity on $X$. More precisely: $Q$ is the Hausdorff dimension of $\partial_{T}$ as well as that of $X ; m_{T}$ is the $Q$-Hausdorff measure on $\partial T ; p$, the integrability exponent; $s$ is the smoothness parameter the parameter; the kernel used to define $\operatorname{Cap}_{\partial T}$ is

$$
K_{T}(x, y)=\left[m_{T}\left(x, \rho_{T}(x, y)\right)+m_{T}\left(y, \rho_{T}(x, y)\right)\right]^{-s}
$$

One of the main difficulties here is that the map $\Lambda$ is not one-to-one, therefore sets having positive capacity in $X$ have multiple preimages, possibly far away, in $\partial T$. This difficulty does not arise when $X$ is homeomorphic to a subset of the real line, since in this case the set of the points having multiple preimages is countable.

Let us mention an immediate consequence of Theorem 1 in the case of Euclidean space $\mathbb{R}^{n}$. The tree of the Euclidean dyadic cubes has a huge group of automorphisms, which are in turn isometries of the corresponding measure-metric structure $\left(T, \rho_{T}, m_{T}\right)$. Such automorphisms are generated by the operation of freely shuffling the $2^{n}$ cubes which are immediately below any given cube in the tree. The capacity of a set is essentially invariant under such automorphism, with multiplicative constants which are independent of the set and of the automorphism. This invariance property is obviously much more general than invariance under isometries of the Euclidean space, although not as sharp. Invariance under tree automorphisms is not obvious, especially in several variables: in higher dimensions portions of the set having positive capacity might be doubled and moved apart one from the other, operations which generally increase capacity.

Here is an outline of the article. In Section 2 we construct the graph $G$ and the tree $T$ associated with $X$, and the surjection $\Lambda: \partial T \rightarrow X$. Among the properties of $\Lambda$ in the section, we show that the natural push-forward of measures $m \mapsto \Lambda_{*} m$ has a (non-natural) right inverse. This allows us to move measures back and forth from $\partial T$ to $X$.

In Section 3 we prove a version of the Muckenhoupt-Wheeden-Wolff inequality for potentials on graphs. The part of the inequality we need is that independently proved by T. Wolff. In Section 4 we show that measures on $\partial T$ and $X$, if they are correspondent under $\Lambda_{*}$ or its inverse map, have comparable energy. As a consequence, we prove Theorem 1.

Section 5 presents the basic facts of potential theory on trees. First, we show that the Muckenhoupt-Wheeden-Wolff inequality establishes a correspondence between "Bessel" potential theory on $X$, hence on $\partial T$, and "logarithmic" potential theory on $T \cup \partial T$. The latter is the sort of potential theory which has been independently developed by several authors over the past twenty years, an account of which is given in the rest of the section. Potential theory on trees is simpler than its counterparts on general Ahlfors-regular spaces for several reasons. The main such reason is that capacities can be computed explicitly by means of 
recursive formulas, which are deduced in the present article in a rather general form. Moreover, in the context of trees, sets' boundaries are often trivial and scaling arguments are elementary and natural. As a consequence, the capacitary potential of a set $E$ is a simple object, whose geometry is linked to that of the set $E$ in a very transparent way.

Some of the material presented here has already appeared in the literature, while some of it is presented here for the first time. We present, in particular, a deduction of the trace inequality for the potential $K$ from a much simpler dyadic "Carleson measure inequality" on trees. Then, we give a rather direct proof that a testing condition known to be equivalent to the trace inequality implies a capacitary condition, also known to be equivalent to the trace inequality. This answers a question Maz'ya asked some of us some years ago.

For ease of the reader, in the Appendix we have translated in tree terms some basic results of Nonlinear Potential Theory, as they are presented in [AH] (Sect. 2.3-2.4).

Our hope is that the simple tree model will be useful to researchers working in or using the results of potential theory. In this paper, we do not offer new applications of the equivalence between "classical" and tree capacities, except for a new proof of the trace inequalities in the Ahlfors-regular case. We hope to return on applications in other papers and that other people will find useful the tool we have here developed.

1.2. Some Examples of Ahlfors-Regular Metric Spaces. We end the introduction by mentioning a few, often not independent, examples of the spaces to which the theory developed in the present paper applies. More material, at a much deeper level, can be found, for example, in [He].

(1) Euclidean $\mathbb{R}^{n}$ and the $n$-dimensional sphere $\Sigma_{n}$ are $n$-dimensional Ahlfors-regular spaces.

(2) Both the closed and open balls in Euclidean space $\mathbb{R}^{n}$ are $n$-regular Ahlfors spaces.

(3) The ternary Cantor set $C$ with the metric inherited from the real line is Ahlforsregular with $Q=\frac{\log 2}{\log 3}$.

(4) If $(X, \rho, m)$ is Ahlfors $Q$-regular, the measure $m$ can be replaced by the $Q$-Hausdorff measure $H_{\rho}^{Q}$ in $(X, \rho)$. The metric measure structure, that is, can be reduced to the metric structure alone.

(5) The "snowflake metric" $\rho(x, y)=|x-y|^{1 / 2}$ makes the real line into an Ahlfors 2regular space.

(6) Carnot groups having homogeneous dimension $Q$ with their Carnot metrics are Ahlfors $Q$-regular. See [BLU] for a comprehensive introduction to the topic.

(7) An $n$-dimensional, complete Riemannian manifold with nonnegative, bounded curvature is Ahlfors $n$-regular.

(8) The unit sphere in $\mathbb{C}^{n}$ endowed with the Koranyi metric $\rho(z, w)=|1-z \cdot \bar{w}|^{1 / 2}$ is an Ahlfors-regular space having dimension $2 n+1$.

(9) If $(X, \rho, m)$ is Ahlfors $Q$-regular and $Q_{\alpha}$ is one of the dyadic boxes in $X$ 's dyadic decomposition (see Christ's Theorem in the next section), then $\bar{Q}_{\alpha}$, the closure of $Q_{\alpha}$ in $X$, is $Q$-regular. See Theorem 8.

(10) The boundary of any homogeneous tree (except $\mathbb{Z}$ ) with respect to the Gromov distance is a regular Ahlfors space. As before, the measure considered here is the $Q$-Haudorff measure, where $Q$ is the Hausdorff dimension of $\partial T$.

Notation. If $A\left(P_{1}, \ldots, P_{n}\right)$ and $B\left(P_{1}, \ldots, P_{n}\right)$ are two positive, or positively infinite, quantities depending on the objects $P_{1}, \ldots, P_{n}$, we write $A \approx B$ if there are constants $0<C_{1}<C_{2}$, 
independent of $P_{1}, \ldots, P_{n}$, s.t $C_{1} A \leq B \leq C_{2} A$. We write $A \lesssim B$ if there is a constant $C>0$ such that $A \leq C B$. We will denote by c a positive constant which might change value within the same expression or calculation.

\section{A Metric Space, a Graph, and a Tree}

In this section we consider the discretization of the Ahlfors-regular space $X$ at different scales by means of metric "dyadic boxes" as proved by M. Christ. While we only need the easy part of Christ's Theorem, we have to be careful: having to deal with Borel measures, we can not discard sets having null m-measure. The difficult part of Christ's is an estimate of the mass concentrated near the boundary of the dyadic boxes. Christ's Theorem can be interpreted as the construction of a graph $G$, having $X$ as boundary. The vertices of the graph are dyadic boxes, which generalize to the context of Ahlfors-regular metric spaces the dyadic decomposition of Euclidean space: each dyadic box is partitioned into a number of smaller dyadic boxes, which we might call its "children". The edges are either "horizontal" or "vertical". Horizontal edges join nearly adjacent dyadic boxes having comparable diameter, while a vertical edge joins a dyadic box with its "parent" dyadic box.

To better clarify this point we bi-Lipschitz modify the original distance $\rho$ to $\bar{\rho}$, a distance which extends to a length-distance on $\bar{G}:=G \cup X$. We then consider the tree structure $T$ of the dyadic boxes: $T$ and $G$ have the same vertices, but $T$ only preserves the vertical edges. The boundary $\partial T$ of $T$ is "larger" than $X$, and can be thought of as a Cantor set. We construct an onto, Lipschitz map $\Lambda: \bar{T} \rightarrow \bar{G}$, mapping boundaries to boundaries and such that $\Lambda(\partial T)=X$. The main point of this section consists in proving that the push-forward of measures $\Lambda_{*} \omega:=\omega \circ \Lambda$ has a (non-canonical) left inverse with nice properties. The main technical difficulty consists in proving that $\Lambda$ maps Borel sets to Borel sets or, rather, a generalization of this fact. Once all this is done, we can move Borel measures back and forth through $\Lambda$.

2.1. Christ's Theorem Revisited. Let $(X, m, \rho)$ be an Ahlfors $Q$-regular metric measure space. We denote the balls by $B(x, r)=\{y \in X: \rho(y, x)<r\}$. In this section we do not assume $X$ to be bounded.

In particular, $(X, \rho)$ is a homogeneous space à la Coifman-Weiss : the measure $m$ satisfies the doubling condition

$$
m(B(x, 2 r)) \leq c_{3} m(B(x, r)) .
$$

It is well known (see, e.g., Semmes' essay in [Gro]) that we can take $m$ to be the $Q$ dimensional Hausdorff measure for the metric space $(X, \rho)$. Namely, if $(X, m, \rho)$ is Ahlfors $Q$-regular and $\mathcal{H}_{\rho}^{Q}$ is the $Q$-dimensional Hausdorff measure for the distance $\rho$, then $\left(X, \mathcal{H}_{\rho}^{Q}, \rho\right)$ is Ahlfors $Q$-regular and there is $A>0$ such that

$$
A^{-1} m(E) \leq \mathcal{H}_{\rho}^{Q}(E) \leq m(E)
$$

for all Borel measurable sets in $X$.

We shall also assume, in the main body of the paper, that $X$ is bounded, $\operatorname{diam}(X) \leq 1$, so that (1) only is required to hold when $r \leq 1$. Under the sole hypothesis that $(X, \rho, m)$ is a homogeneous space, M. Christ [Christ] proved that $X$ admits a "dyadic" decomposition. We state here the easy part of Christ's Theorem.

Theorem A. [M. Christ] 
There exists a collection $\left\{Q_{\alpha}^{k}, \alpha \in I_{k}, k \in \mathbb{Z}\right\}$ of open subsets of $X$ and $\delta>0, a_{0}>0$, $c_{4}>0$ such that

(i) $m\left(X \backslash \cup_{\alpha \in I_{k}} Q_{\alpha}^{k}\right)=0$ holds for all $k \geq 0$;

(ii) if $l \geq k$ then, for all $\alpha \in I_{k}$ and $\beta \in I_{l}$, either $Q_{\beta}^{l} \subseteq Q_{\alpha}^{k}$, or $Q_{\beta}^{l} \cap Q_{\alpha}^{k}=\emptyset$;

(iii) for all $(l, \beta)$ and $l>k$, there is a unique $\alpha$ in $I_{k}$ such that $Q_{\beta}^{l} \subseteq Q_{\alpha}^{k}$;

(iv) $\operatorname{diam}\left(Q_{\alpha}^{k}\right) \leq c_{4} \delta^{k}$;

(v) for all $(k, \alpha), B\left(z_{\alpha}^{k}, \delta^{k}\right) \subseteq Q_{\alpha}^{k}$, for distinguished points $z_{\alpha}^{k} \in Q_{\alpha}^{k}$.

If $X$ is bounded, after rescaling the distance we can assume that $k \in \mathbb{N}$ and that $I_{0}$ contains a unique element $o: Q_{o}^{0}=X$. By analogy with the case of Euclidean space, we will call the sets $Q_{\alpha}^{k}$ or their closures dyadic sets or qubes.

We take the sets $\alpha \equiv(k, \alpha) \leftrightarrow Q_{\alpha}^{k}$ themselves as points of a new space $T$. When we do not want to stress the level of $\alpha$, we simply write $Q_{\alpha}$ instead of $Q_{\alpha}^{k}$.

For $\alpha \in I_{k}$, we set $k=d(\alpha)$ to be the level of $\alpha$. The set $T$ is given a tree structure: there is an edge of the tree between $\alpha$ and $\beta$ if $d(\beta)=d(\alpha)+1$ and $\beta \subseteq \alpha$, possibly interchanging the rôles of $\alpha$ and $\beta$. The tree $T$ has a natural, edge-counting distance $d$, which is realized by geodesics. We introduce the partial order: $\alpha \leq \beta$ in $T$ if $\alpha \in[o, \beta]$, the geodesic joining $o$ and $\beta$.

We also introduce on $T$ a further graph structure: two distinct points $\alpha$ and $\beta$ in $T$ are connected by an edge of the graph $G$ if they are already connected by an edge of $T$, or if $d(\alpha)=d(\beta)=k$ and there are points $x \in \alpha$ and $y \in \beta$ such that $\rho(x, y) \leq \delta^{k}$. In this case, we write $\alpha_{G}^{\sim} \beta$.

The natural edge-counting distance in $G$ is denoted by $d_{G}$. It is realized by geodesics, but, contrary to the tree case, there might be several geodesics joining two points.

We identify $T=G$ as sets of vertices, and use different names when dealing with different edge structures. It would be more elegant to use two names for the different edge sets and one third name for the common vertex set. We have here sacrified elegance to economy, more in line with the dialect of "dyadic" analysts than with the language of graph theorists.

In Graph Theory it is said that $T$ is a spanning tree for the graph $G$.

Since we are interested in possibly singular measures on $X$, we have to refine Christ's construction a little in order to have regions whose union gives us back the whole space $X$. This is done in the following lemmas.

Lemma 2. For each $k$, we have that $\cup_{\alpha \in I_{k}} \overline{Q_{\alpha}^{k}}=X$.

Proof. By contradiction, suppose that $x$ lies in $X \backslash \cup_{\alpha \in I_{k}} \overline{Q_{\alpha}^{k}}$. Then, any ball $B(x, \epsilon)$ intersects $Q_{\alpha}^{k}$ for infinitely many $\alpha$ in $I_{k}$. In fact, since $m(B(x, \epsilon))>0$, by (i) in Theorem A and the assumption that open sets have, by Ahlfors-regularity, positive measure, there exists $\alpha_{1}$ in $I_{k}$ such that $B(x, \epsilon) \cap Q_{\alpha_{1}}^{k} \neq \emptyset$. There must be some $0<\epsilon_{2}<\epsilon=\epsilon_{1}$ such that $B\left(x, \epsilon_{2}\right) \cap Q_{\alpha_{1}}^{k}=\emptyset$, otherwise $x \in \overline{Q_{\alpha_{1}}^{k}}$, a contradiction. As before, there must be then $\alpha_{2} \neq \alpha_{1}, \alpha$ such that $B\left(x, \epsilon_{2}\right) \cap Q_{\alpha_{2}}^{k} \neq \emptyset$.

Iterating this procedure, we find a sequence $Q_{\alpha_{n}}^{k}$ of distinct regions at generation $k$. They have to be disjoint by (ii) in Christ's Theorem. Also, they are contained in the ball $B(x, \epsilon+$ $2 c_{4} \delta^{k}$ ), by (iv), and each of them has volume at least $c_{1} \delta^{Q k}$, by Ahlfors-regularity (1). Hence,

$$
+\infty=\sum_{n} m\left(Q_{\alpha_{n}}^{k}\right)=m\left(\cup_{n} Q_{\alpha_{n}}^{k}\right) \leq m\left(B\left(x, \epsilon+2 c_{4} \delta^{k}\right)\right)<+\infty,
$$

a contradiction. 
Lemma 2 is trivial if $\operatorname{diam}(X)<\infty$, having in this case $I_{k}$ finitely many elements only. From the lemma, the fact that the regions $Q_{\alpha}^{k}$ are open, and the regularity of the measure $m$, we deduce immediately the following.

Corollary 3. Let $F^{k}=\cup_{\alpha \in I_{k}} \partial Q_{\alpha}^{k}$ and $F=\cup_{k} F^{k}$. Then, each $F^{k}$ is closed, $F^{k} \subseteq F^{k+1}$ and $m(F)=0$.

Proof. We first show that $F^{k}$ is closed. Let $x_{n} \in \partial Q_{\alpha_{n}}^{k}, x_{n} \rightarrow x$ in $X$. By Lemma 2, if $x \notin F^{k}$, then $x \in Q_{\alpha}^{k}$ for some $\alpha \in I_{k}$, but $Q_{\alpha}^{k}$ is open and the the $Q_{\beta}^{k}$ 's are disjoint, hence $x_{n} \notin F^{k}$ for large $n$ : a contradiction.

The inclusion $F^{k} \subseteq F^{k+1}$ easily follows from Lemma 2 and (iii) in Christ's Theorem.

Assertion (i) in Theorem 2.1 implies that $m\left(F^{k}\right)=0$, and $m(F)=0$ by regularity of the measure $m$.

\section{Lemma 4.}

(i) There exists $c_{5}$ independent of $k \geq 0$ and of $x \in X$ such that $\sharp\left\{\alpha \in I_{k}: x \in \overline{Q_{\alpha}^{k}}\right\} \leq c_{5}$;

(ii) There exists $c_{6}$ independent of $\alpha$ in $G$ such that $\sharp\left\{\beta \in G: \beta_{G}^{\sim} \alpha\right\} \leq c_{6}$ : the graph $G$ has bounded degree.

(iii) In particular, the number of $\beta \in I_{k+1}$ such that $Q_{\beta}^{k+1}$ is contained in a fixed $Q_{\alpha}^{k}$ is bounded by a constant $c$ which only depends by the structural constants of the metric space $(X, \rho)$.

Proof. It is clear that (i) is a consequence of (ii). In fact, all $\alpha \in I_{k}$ for which $x$ lies in $\overline{Q_{\alpha}^{k}}$ are $G$-related.

Fix $x \in Q_{\alpha}^{k}$ and suppose that $\beta_{G}^{\sim} \alpha$. Consider first the case $\beta \in I_{k}$. There is then a constant $c$ such that, if $\rho\left(Q_{\alpha}^{k}, Q_{\beta}^{k}\right) \leq \delta^{Q}$, then $Q_{\beta}^{k} \subseteq B\left(x, c \delta^{k}\right)$. Now, using Ahlfors $Q$-regularity and items (iv), (v) of Christ's Theorem in the first inequality,

$$
\begin{aligned}
\delta^{Q k} \cdot \sharp\left\{\beta \in I_{k}: Q_{\beta}^{k} \cap B\left(x, \delta^{k}\right) \neq \emptyset\right\} & \leq c \sum_{Q_{\beta}^{k} \cap B\left(x, \delta^{k}\right) \neq \emptyset} m\left(Q_{\beta}^{k}\right) \\
& =m\left(\cup_{Q_{\beta}^{k} \cap B\left(x, \delta^{k}\right) \neq \emptyset} Q_{\beta}^{k}\right) \\
& \leq m\left(B\left(x, c \delta^{k}\right)\right) \leq c \delta^{Q k},
\end{aligned}
$$

which implies (ii) under the restriction $\beta \in I_{k}$. The general case easily follows from the special one and Ahlfors-regularity.

Lemma 5. If $\alpha \in I_{k}$ and $h \geq 0$, then

$$
\bar{Q}_{\alpha}=\cup_{\beta \geq \alpha, \beta \in I_{k+h}} \bar{Q}_{\beta} .
$$

Proof. Clearly, $\bar{Q}_{\alpha} \supseteq \cup_{\beta \geq \alpha, \beta \in I_{k+h}} \bar{Q}_{\beta}$. In the other direction, since the $Q_{\beta}$ 's are finitely many, it suffices to show that $\cup_{\beta \geq \alpha, \beta \in I_{k+h}} \bar{Q}_{\beta}$ is dense in $Q_{\alpha}$. If this were not the case, there would be a metric ball $B(z, \epsilon)$ in $Q_{\alpha} \backslash\left[\cup_{\beta \geq \alpha, \beta \in I_{k+h}} \bar{Q}_{\beta}\right]$, hence, by Ahlfors-regularity,

$$
m\left(Q_{\alpha}\right)>\sum_{\beta \geq \alpha, \beta \in I_{k+h}} m\left(Q_{\beta}\right),
$$

which, (i) and (iii) in the Theorem of Christ, contradicts Corollary 3.

Complete Ahlfors-regular spaces are not especially exotic among metric spaces. 
Corollary 6. $(X, \rho)$ is locally compact.

Proof. Mimic the proof of the Heine-Borel Theorem in [Ru], p.38-40 using the dyadic decomposition of Christ instead of the usual dyadic decomposition of $\mathbb{R}^{n}$, and the metric completeness of $X$ instead of the completeness of $\mathbb{R}$.

Corollary 7. $(X, \rho)$ is separable.

Proof. It suffices to show that each $\bar{Q}_{\alpha}$ is separable. By Christ Theorem, for each integer $n>0$, there are finitely many points $\left\{z_{j}^{n}: j=1, \ldots, k_{n}\right\}$ in $\bar{Q}_{\alpha}$ such that any point $x$ in $\bar{Q}_{\alpha}$ lies at distance at most $2^{-n}$ from some $z_{j}^{n}$. The countable set $\left\{z_{j}^{n}: j=1, \ldots, k_{n}, n \geq 1\right\}$ is dense in $\bar{Q}_{\alpha}$.

Theorem 8. Each set $X_{\alpha}=\bar{Q}_{\alpha}$ is Ahlfors $Q$-regular. Moreover, the dyadic sets in Christ's decomposition of $X_{\alpha}$ might be taken to be the sets $Q_{\beta}$ where $\beta \geq \alpha$.

Proof. Assume without loss of generality that $\alpha \in I_{0}$ and let $x$ be a point of $\bar{Q}_{\alpha}$. Let $r \in$ $(0,1), \delta^{k+1}<r \leq \delta^{k}$, and let $\beta \in I_{k}, \beta \geq \alpha$, be such that $x \in \bar{Q}_{\beta}$ (at least one such $\beta$ exists by Lemma 5). By Christ's Theorem, there is constant $C$ such that $Q_{\alpha} \cap B\left(x, C \delta^{k}\right) \supseteq Q_{\beta}$, hence,

$$
m\left(Q_{\alpha} \cap B\left(x, C \delta^{k}\right)\right) \geq m\left(Q_{\beta}\right) \geq C^{\prime} \delta^{Q k} .
$$

Ahlfors-regularity of $\bar{Q}_{\alpha}$ immediately follows.

2.2. The space $X$ as the Boundary of $G$. We now define a new distance $\bar{\rho}$ on $G \cup X$. Let $\alpha \neq \beta$ be points of $G$ such that $d(\alpha) \leq d(\beta) \leq d(\alpha)+1$ and $\alpha_{G}^{\sim} \beta$.

To the edge $[\alpha, \beta]$ we associate the weight $\ell_{G}([\alpha, \beta]):=\delta^{d(\alpha)}$. The length of a path $\Gamma=\left(\alpha_{0}, \ldots, \alpha_{n}\right)$ in $G$ is defined by adding the length of its edges.

If needed, we set $\ell_{G}((\alpha))=0$, where $(\alpha)$ is the trivial path from $\alpha$ to itself. The distance $\bar{\rho}$ is defined, on points $\alpha, \beta \in G$, as

$$
\bar{\rho}(\alpha, \beta)=\inf \left\{\ell_{G}(\Gamma): \Gamma \text { a path having endpoints } \alpha \text { and } \beta\right\} .
$$

By the triangle inequality,

$$
\rho\left(Q_{\alpha}, Q_{\beta}\right) \leq c \bar{\rho}(\alpha, \beta) .
$$

Let $(\bar{G}, \bar{\rho})$ be the completion of the metric space $(G, \bar{\rho})$. By this we mean the usual Cantor construction: Cauchy sequences $\left\{\alpha_{n}\right\}$ and $\left\{\beta_{n}\right\}$ in $(G, \rho)$ are identified if $\lim _{n \rightarrow \infty} \rho\left(\alpha_{n}, \beta_{n}\right)=$ 0 . Each point $\alpha \in G$, identified with the equivalence class of the sequences in $G$ taking value $\alpha$ definitely, is isolated in $\bar{G}$. Let $\partial G:=\bar{G} \backslash G$. There is a bijection b between $\partial G$ and $X$.

The metric space $\bar{G}$ is the Floyd completion of $G$. We will show in Theorem 10 that the map $b$ is bi-Lipschitz and onto. A more general result is stated in Theorem 4.7 of the very informative [Geo]. It is also shown there that the weights can be chosen in such a way $b$ becomes an isometry. We thank the referee for making us aware of the article [Geo], that also contains a detailed description of much related literature. In order to keep the exposition as self-contained as possible, and close to the viewpoint given by Christ's Theorem, we provide our own proof of Theorem 10. 
Lemma 9. Let $a=\left\{\alpha_{n}\right\}$ be Cauchy sequence in $(G, \bar{\rho})$, which is not definitely constant, and let $[a]$ be the equivalence class of a in $\partial G$. Define

$$
b([a])=x \in X
$$

if and only if $\lim _{n \rightarrow \infty} \rho\left(x, Q_{\alpha_{n}}\right)=0$. Then, $b$ is a well defined bijection of $\partial G$ onto $X$.

Proof. The proof will be broken up into several steps.

- To each Cauchy sequence b associates a unique $x \in X$.

Let $d\left(\alpha_{n}\right)$ be the level of $\alpha_{n}$ in $T$. Since the sequence is not definitely constant, it is Cauchy and $G$ is discrete, we must have $\lim _{n \rightarrow \infty} d\left(\alpha_{n}\right)=+\infty$. Let $z_{n}$ be a distinguished point in $Q_{\alpha_{n}}$. Let $n, m \geq 1$. Using (7),

$$
\begin{aligned}
\rho\left(z_{n}, z_{n+m}\right) & \leq \operatorname{diam}\left(Q_{\alpha_{n}}\right)+\rho\left(Q_{\alpha_{n}}, Q_{\alpha_{n+m}}\right)+\operatorname{diam}\left(Q_{\alpha_{n+m}}\right) \\
& \leq c\left(\delta^{d\left(\alpha_{n}\right)}+\bar{\rho}\left(\alpha_{n}, \alpha_{n+m}\right)+\delta^{d\left(\alpha_{n+m}\right)}\right) \rightarrow 0,
\end{aligned}
$$

as $n \rightarrow \infty$. Since $(X, \rho)$ is complete and $\left\{z_{n}\right\}$ is Cauchy, it has a well defined limit $x$ and $\lim _{n \rightarrow \infty} \rho\left(x, Q_{\alpha_{n}}\right) \leq \lim _{n \rightarrow \infty} \rho\left(x, z_{n}\right)=0$. This shows existence of $x$.

Being the sequence $\alpha_{n}$ non-definitely constant, the $\rho$-diameter of $Q_{\alpha_{n}}$ tends to 0 as $n \rightarrow \infty$, which immediately implies the uniqueness of $x$ with the property . $\lim _{n \rightarrow \infty} \rho\left(x, Q_{\alpha_{n}}\right)=0$.

- If $[a]=[b]$, $a$ and $b$ define the same point in $X: b([a])=b([b])$.

Let $a=\left\{\alpha_{n}\right\}$ and $b=\left\{\beta_{n}\right\}$. Choose distinguished points $z_{n} \in Q_{\alpha_{n}}$ and $w_{n} \in Q_{\beta_{n}}$. Reasoning as above, $\lim _{n \rightarrow \infty} \rho\left(z_{n}, w_{n}\right)=0$, hence the two sequences converge to the same point $x \in X$.

- $b: \partial G \rightarrow X$ is surjective.

Given $x$ in $X$, for each $n \geq 0$ we can choose by Lemma 2 a $Q_{\alpha_{n}}^{n}$ such that $x \in \overline{Q_{\alpha_{n}}^{n}}$. It is easily checked that the graph distance between $\left(n, \alpha_{n}\right)$ and $\left(n+1, \alpha_{n+1}\right)$ is at most 2 and that $b\left(\left[\left\{\left(n, \alpha_{n}\right)\right\}\right]\right)=x$.

- $b$ is injective. Suppose that $a=\left\{\alpha_{n}\right\}, b=\left\{\beta_{n}\right\}$ are Cauchy sequences in $(G, \bar{\rho})$, not definitely constant, and that $b([a])=b([b])$. This means that there is $x \in X$ is such that $\lim _{n \rightarrow \infty} \rho\left(Q_{\alpha_{n}}, x\right)=\lim _{n \rightarrow \infty} \rho\left(Q_{\beta_{n}}, x\right)=0$. Since the diameters of $Q_{\alpha_{n}}$ and $Q_{\beta_{n}}$ tend to zero and, by the triangle property, $\rho\left(Q_{\alpha_{n}}, Q_{\alpha_{n}}\right)$ tends to zero, $[a]=[b]$.

The map $b$ in Lemma 9 identifies the metric measure space $X$ we started with the metric boundary of a graph $G$. From now on, we simply write $\partial G=X$. The theorem below says that such identification carries all the important metric structure of $X$.

\section{Theorem 10.}

(i) The metric $\bar{\rho}$, restricted to $X$, is bi-Lipschitz equivalent to $\rho$ :

$$
c \rho(x, y) \leq \bar{\rho}(x, y) \leq c \rho(x, y) .
$$

(ii) Let $Z_{k}=\left\{z_{\alpha}^{k}: \alpha \in I_{k}\right\}$ be the set of the distinguished points $z_{\alpha}^{k} \in Q_{\alpha}^{k}$ mentioned in Theorem A; and let $G_{k}=\{\alpha \in G: d(\alpha)=k\}$. Then, $\left.\rho\right|_{Z_{k}}$ is bi-Lipschitz equivalent to $\left.\bar{\rho}\right|_{G_{k}}$,

$$
c \rho\left(z_{\alpha}^{k}, z_{\beta}^{k}\right) \leq \bar{\rho}(\alpha, \beta) \leq c \rho\left(z_{\alpha}^{k}, z_{\beta}^{k}\right) .
$$


The theorem says that all Ahlfors-regular spaces (bounded and complete, so far, but we think that these further assumptions can be easily removed) are - modulo a bi-Lipschitz change of the metric - the boundaries of rather regular and concrete graphs. Or, in other terms, that all such metric spaces admit a natural extension to a metric measure space in which the distance is given by length of curves and it is realized, it can be shown, by geodesics. The theme of geodesics is discussed in depth in[Geo].

By fattening the graph's edges, we might even see that the space $X=\partial G$ is the metric boundary (modulo bi-Lipschitz change of metric) of a Riemannian manifold $M$ which maintains many of the properties of $X$. $M$, for instance, could be taken to be Ahlfors regular, having as topological and Hausdorff dimension the least integer $n>Q$.

Proof. (i) Let $x, y$ be points in $X$. We show first that $\bar{\rho}(x, y) \leq c \rho(x, y)$. Suppose that $\rho(x, y) \leq \delta^{k}$ and let $\alpha, \beta \in I_{k}$ be such that $x \in \overline{Q_{\alpha}^{k}}, y \in \overline{Q_{\beta}^{k}}$. Then, there is an edge of $G$ between $\alpha$ and $\beta$, hence $\bar{\rho}(\alpha, \beta) \leq \delta^{k}$. Arguing like in the proof of b's surjectivity, we can find sequences $a=\left\{\alpha_{n}\right\}$ and $b=\left\{\beta_{n}\right\}$ such that $\alpha_{0}=\alpha, \beta_{0}=\beta ; \beta_{n}, \alpha_{n} \in I_{k+n} ; x \in \overline{Q_{\alpha_{n}}}$ and $y \in \overline{Q_{\beta_{n}}}$. Two successive elements of the sequence $a$ have graph distance at most 2 , and the same holds for $b$. A geometric series applied to the sequences shows then that $\bar{\rho}(\alpha, x) \leq c \delta^{k}$ and $\bar{\rho}(y, \beta) \leq c \delta^{k}$. Then, $\bar{\rho}(x, y) \leq c \delta^{k}$, as wished.

In the other direction, assume that $\rho(x, y)>0$ (otherwise there is nothing to prove), with $x=b\left(\left[\left\{\alpha_{n}\right\}\right]\right)$ and $y=b\left(\left[\left\{\beta_{n}\right\}\right]\right)$. By Lemma 9 , for each $\epsilon>0$ we can take $n=n(\epsilon)$ large enough to have:

(i) $\bar{\rho}\left(x, \alpha_{n}\right), \bar{\rho}\left(y, \beta_{n}\right) \leq \epsilon \rho(x, y)$ (because $\left\{\alpha_{n}\right\}$ and $\left\{\beta_{n}\right\}$ are Cauchy sequences tending to $x$ and $y$, respectively, in $\bar{G})$;

(ii) $\rho\left(x, \alpha_{n}\right), \rho\left(y, \beta_{n}\right) \leq \epsilon \rho(x, y)$ (by definition of $b$ );

(iii) $n \geq n_{0}$ to be chosen.

Let $\left[\gamma_{0}=\alpha_{n}, \gamma_{1}, \ldots, \gamma_{m}=\beta_{n}\right]$ be any path in $G$ which joins $\alpha_{n}$ and $\beta_{n}$, and choose distinguished points $w_{j} \in Q_{\gamma_{j}}(o \leq j \leq m)$. We have:

$$
\begin{aligned}
\rho(x, y) & \leq \rho\left(x, w_{0}\right)+\sum_{j=1}^{m} \rho\left(w_{j}, w_{j-1}\right)+\rho\left(w_{m}, y\right) \\
\leq & 2 \epsilon \rho(x, y)+\operatorname{diam}\left(Q_{\alpha_{n}}\right)+\operatorname{diam}\left(Q_{\beta_{n}}\right) \\
& +\sum_{j=1}^{m} \rho\left(w_{j}, w_{j-1}\right)+\rho\left(w_{m}, y\right) \\
& \text { by }(\mathrm{ii}) \\
\leq & 4 \epsilon \rho(x, y)+c \ell_{G}\left(\left[\gamma_{o}, \ldots, \gamma_{m}\right]\right),
\end{aligned}
$$

by (iii) and by definition of $\ell_{G}$. Passing to the infimum of the lengths,

$$
\begin{aligned}
(1-4 \epsilon) \rho(x, y) & \leq c \bar{\rho}\left(\alpha_{n}, \beta_{n}\right) \\
& \leq c\left[\bar{\rho}(x, y)+\bar{\rho}\left(x, \alpha_{n}\right)+\bar{\rho}\left(y, \beta_{n}\right)\right] \\
& \leq c \bar{\rho}(x, y)+2 c \epsilon \rho(x, y)
\end{aligned}
$$

by (i). It suffices to choose $\epsilon$ small enough to get the desired inequality.

Part (ii) of the theorem is proved similarly. 
A consequence of the proof is that, given $x, y \in X$, there exists a "near geodesic" in $\bar{G}$ for the distance $\bar{\rho}(x, y)$, passing through a point at level $k$, where $\rho(x, y) \approx \delta^{k}$. This fact, indeed, extends the well known relations between Euclidean and hyperbolic distances, and geodesics, in the upper half space.

When the space $(X, \rho)$ has a metric defined by a length, like the spaces introduced by Heinonen and Koskela in [HeK], then we find "near geodesics" joining $x, y \in X$ at all levels $n$ of $\bar{G}$ such that $\delta^{n} \leq \rho(x, y)$.

When the space is poor of rectifiable curves, as it happens with "snowflake" metrics on the real line, the near geodesics joining $x$ and $y$ cannot be completely contained in a small strip of $\bar{G}$ : they have to reach a level $n$ such that $\delta^{n} \gtrsim \rho(x, y)$.

\subsection{The Tree and its Boundary as Metric Spaces.}

2.3.1. The Boundary of a Tree. Let $T$ be a tree with root $o$ (not necessarily the tree arising from Christ's Theorem). We assume that $T$ has bounded degree and that it has no childless vertices: there is $N \geq 1$ such that each vertex $\alpha$ in $T$ has $N(\alpha)$ children, with $1 \leq N(\alpha) \leq N$.

We introduce some notation which will be frequently used. If $\alpha$ is a vertex of $T$, the predecessor set of $\alpha$ is $P(\alpha)=[o, \alpha]$, while the successor set of $\alpha$ is $S(\alpha)=\{\beta \in T: \alpha \in$ $P(\beta)\}$. Given $\alpha$ and $\beta$ in $T, \alpha \wedge \beta=\max (P(\alpha) \cap P(\beta))$ is their confluent. The predecessor $\alpha^{-1}$ of $\alpha \in T \backslash\{o\}$ is the only element $\beta$ in $P(\alpha)$ such that $d(\alpha, \beta)=1$.

The combinatorial boundary $\tilde{\partial} T$ of $T$ is the set of all half-infinite geodesics, with respect to the edge counting distance, having an endpoint at the root. For ease of notation, we think of $\tilde{\partial} T$ as a set of labels $\zeta$ of the geodesics $\Gamma_{\zeta}$. The topology for $\tilde{\partial} T$ is that having as basis the sets

$$
\partial S(\alpha)=\left\{\zeta \in \tilde{\partial} T: \alpha \in \Gamma_{\zeta}\right\}
$$

Let now $\delta \in(0,1)$ be fixed. Associate to each edge $\left(x^{-1}, x\right)$ of $T\left(x^{-1}\right.$, we recall, is the parent of $x$ ) the weight $w\left(x, x^{-1}\right)=\delta^{d(x, o)}$, where $d$ is the edge-counting distance in $T$.

The metric $\rho_{T}$ on $T$ with parameter $\delta$ is defined as the length-metric associated with the weight $w$. The geodesics for $\rho_{T}$ are clearly the same as the geodesics for the edge-counting metric. Actually, the poverty of the tree structure is such that all length-metrics have the same geodesics; and the edge-counting metric is a particular length metric. Let $\bar{T}$ be the metric completion of $T$ with respect to $\rho_{T}$. The metric boundary $\partial T$ of $T$ is $\bar{T} \backslash T$, with the topology induced by the metric $\rho_{T}$.

Lemma 11. For each $\zeta$ in $\tilde{\partial} T$, consider the geodesic $\Gamma_{\zeta}$ as a sequence of points in $T$. Then,

(i) $\Gamma_{\zeta}$ is a Cauchy sequence for $\rho_{T}$;

(ii) Let $\downarrow(\zeta)$ be the equivalence class of $\Gamma_{\zeta}$ in $\partial T$. The map $\zeta \mapsto \downarrow(\zeta)$ is a homeomorphism of $\tilde{\partial} T$ onto $\partial T$.

The proof of the lemma is easy and it is left to the reader.

Extend $\downarrow$ to a map from $T \cup \tilde{\partial} T$ to $T \cup \partial T$ by letting $\left.\sharp\right|_{T}=\left.I d\right|_{T}$. Requiring the extended $\natural$ to be a homeomorphism gives a topological structure to $\bar{T}=T \cup \tilde{\partial} T$.

We extend the tree notation to take into account the boundary. If $\zeta \in \partial T$, let $P(\zeta)=\Gamma_{\zeta}$ is the geodesic from the root to $\zeta$.

For $\alpha, \beta \in \bar{T}$, let

$$
\alpha \wedge \beta=\max (P(\alpha) \cap P(\beta))
$$


be the confluent of $\alpha$ and $\beta$. Observe that the geodesic $[\alpha, \beta]$ between $\alpha$ e $\beta$ in $\bar{T}$ with respect to the metric $\rho_{T}$ is

$$
[\alpha, \beta]=[P(\alpha) \cup P(\beta)] \backslash P\left((\alpha \wedge \beta)^{-1}\right),
$$

thinking of geodesics as sets of vertices, rather than sequences of adjacent edges.

If $\alpha$ is an element of $T, \partial S(\alpha) \subseteq \partial T$ is the set of the half-infinite geodesics passing through $\alpha: \partial S(\alpha)=\overline{S(\alpha)} \backslash S(\alpha)$. The metric $\rho_{T}$ can be explicitly computed. If $\zeta, \xi \in \bar{T}$, then

$$
\rho_{T}(\zeta, \xi)=\frac{2 \delta}{1-\delta}\left[\delta^{d(\zeta \wedge \xi)}-\frac{1}{2}\left(\delta^{d(\zeta)}+\delta^{d(\xi)}\right)\right] .
$$

In some references $\rho_{T}$ is called the Gromov metric on $\bar{T}$. The restriction of $\rho_{T}$ to $\partial T$ is

$$
\rho_{T}(\zeta, \xi)=\frac{2 \delta}{1-\delta} \delta^{d(\zeta \wedge \xi)}
$$

which is called ultrametric in another stream of the vast literature concerning metric spaces.

The balls in $\left(\partial T, \rho_{T}\right)$ are exactly the cl-open sets $\partial S(\alpha)$ and $T$ itself might be seen as the tree of the metric balls in $\partial T$.

2.3.2. The tree $T$ associated with the graph $G$. Let now $T$, the rooted tree, and $\delta>0$ be the same as in Christ's Theorem. The metric $\rho_{T}$ just defined on $\bar{T}$ is the length-metric associated with the length $\ell_{T}$, which is the restriction of the length $\ell_{G}$ to the edges of $T$. The trivial estimate $\rho_{T}(\alpha, \beta) \geq \bar{\rho}(\alpha, \beta)$ cannot in general be reversed.

We can define a Borel measure $\tilde{m}$ on $\partial T$ by declaring that

$$
\tilde{m}(\partial S(\alpha)):=m\left(Q_{\alpha}\right) \text {. }
$$

It is easy to see that the definition is consistent (by Christ's Theorem).

Proposition 12. The space $\left(\partial T, \rho_{T}, \tilde{m}\right)$ is complete and Ahlfors $Q$-regular.

The statement follows immediately from the fact noted above, that the metric balls in $\partial T$ are the sets of the form $\partial S(\alpha)$.

2.4. The Map $\Lambda: \partial T \rightarrow X$. Let $P_{T}(\xi)=\left(\xi_{n}\right)$ be the geodesic starting at the root $o$ and ending at the boundary point $\xi \in \partial T$ (we might, and sometimes will, identify $\xi \equiv P_{T}(\xi)$ ). Each $\xi_{n} \in T$ appearing in $P_{T}(\xi)$ can be identified with a dyadic box $Q\left(\xi_{n}\right)$ in $I_{n}$.

We define a map $\Lambda: \partial T \rightarrow X$,

$$
\Lambda: \xi \mapsto \Lambda(x)=\cap_{n \geq 0} \overline{Q\left(\xi_{n}\right)} .
$$

By the discussion above, $\Lambda$ is a contraction,

$$
\bar{\rho}(\Lambda(\zeta), \Lambda(\xi)) \leq \rho_{T}(\zeta, \xi) .
$$

The map $\Lambda$ is not open, however, neither is it one-to-one, in general.

Lemma 13. There is a constant $c$ independent of $x$ such that

$$
\sharp\{\zeta \in \bar{T}: \Lambda(\zeta)=x\} \leq c .
$$

Proof. Let $\zeta^{(1)}, \ldots, \zeta^{(n)}$ be points in $\partial T$ such that $\Lambda\left(\zeta^{(j)}\right)=x, \zeta^{(j)}=\left(\zeta_{k}^{(j)}\right)_{k \in \mathbb{N}}$. Let $N=\max \left\{d\left(\zeta^{(j)} \wedge \zeta^{(i)}\right): i \neq j=1, \ldots, n\right\}$. Then, $x \in \overline{\zeta_{N+1}^{(1)}} \cap \cdots \cap \overline{\zeta_{N+1}^{(n)}}$, and the dyadic sets $\overline{\zeta_{N+1}^{(j)}}$ are all distinct. By Lemma 4 (i), $n \leq c_{5}$. 
Lemma 14. $\Lambda$ maps $\partial T$ onto $X$.

Proof. Let $x$ be a point of $X, k \geq 0$, consider those $Q_{\alpha}^{k}$ such that $x \in \overline{Q_{\alpha}^{k}}$, and let $J_{k}$ be their set. Each dyadic set in $J_{k+1}$ must have its parent in $J_{k}$ : if $x \in Q_{\alpha}^{k+1}$, this is obvious; if $x \in \partial Q_{\alpha}^{k+1}$, and $Q_{\beta}^{k}$ is the parent of $Q_{\alpha}^{k+1}$, then $x \in \overline{Q_{\beta}^{k}}$. This shows that $J=\cup_{k} J_{k}$ is a full subtree of $T: o \in J$ and $\alpha, \beta \in J \Longrightarrow[\alpha, \beta] \subseteq J . J$ has elements at each level $k \geq 0$ and it has bounded degree. By König's Infinity Lemma in graph theory, $J$ contains an infinite geodesic $\zeta$, and clearly $\Lambda(\zeta)=x$. The last assertion could also be deduced directly from the Axiom of Choice.

At this point, we can be more explicit about the shape of our "near geodesics". Let $x \neq y \in X$ and let $k \geq 0$ an integer such that $\delta^{k+1} \leq \rho(x, y) \leq \delta^{k}$. Consider tree geodesics $\Gamma_{x}, \Gamma_{y}$ starting at level $k$ and going all the way down to $x, y$ respectively, and let $\gamma_{k}(x)$ and $\gamma_{k}(y)$ be their points at level $k$. Clearly, $\rho\left(Q_{\gamma_{k}(x)}, Q_{\gamma_{k}(y)}\right) \leq c \delta^{k}$. Hence, there is a path $\Gamma^{\prime}$ in $G$, having length $\ell_{G}\left(\Gamma^{\prime}\right) \lesssim \delta^{k}$ joining $\gamma_{k}(x)$ and $\gamma_{k}(y)$. Furthermore, there is a fixed constant $h \in \mathbb{N}$ such that the path $\Gamma^{\prime}$ consists of at most $h$ edges between level $k$ and level $k-h$. The path $\Gamma_{x} \cup \Gamma^{\prime} \cup \Gamma_{y}$ is clearly a near geodesic between $x$ and $y$.

2.5. Moving Measures through $\Lambda$. We can use the map $\Lambda$ to push Borel measures from $\partial T$ to $X$. If $\nu$ is a measure on $\partial T$, let

$$
\Lambda_{*} \nu(E):=\nu\left(\Lambda^{-1}(E)\right),
$$

whenever $E$ is Borel measurable in $X$. We want to move measures in the other direction, as well. Let $\omega$ be a nonnegative, Borel measure on $X$, and -for each Borel subset $A$ of $\partial T$ and $x \in X$, set

$$
N(x)=\sharp\{\zeta \in \partial T: \Lambda(\zeta)=x\}, N_{A}(x)=\sharp\{\zeta \in A: \Lambda(\zeta)=x\},
$$

and define

$$
\Lambda^{*} \omega(A):=\int_{X} \frac{N_{A}(x)}{N(x)} d \omega(x) .
$$

Note that, contrary to the pushforward $\Lambda_{*}$, the operator $\Lambda^{*}$ is non canonical. The integral (9) is well defined because $x \mapsto N_{A}(x)$ is Borel measurable, a fact which will be proved below.

For each $x$ in $X$, let $\nu_{x}(A)=\frac{\sharp\left(\Lambda^{-1}(x) \cap A\right)}{\sharp\left(\Lambda^{-1}(x)\right)}$ be the normalized counting measure on $\Lambda^{-1}(x)$. Then

$$
\int_{\partial T} \varphi(\zeta) d \Lambda^{*} \omega(\zeta)=\int_{X}\left\{\int_{\Lambda^{-1}(x)} \varphi(\zeta) d \nu_{x}(\zeta)\right\} d \omega(x)
$$

whenever $\omega$ is a Borel measure on $X$ and $\varphi$ is Borel measurable.

We need the following lemma, whose proof is rather tedious, but reveals the inner functioning of the map $\Lambda$.

Lemma 15. Each Borel set $A$ in $\partial T$ can be decomposed $A=\coprod_{i} A_{i}$ as the disjoint, countable union of Borel sets $A_{i}$ such that the restriction $\left.\Lambda\right|_{A_{i}}$ of $\Lambda$ to each $A_{i}$ is $1-1$. Moreover, if $A$ is a Borel set in $\partial T$. Then, $\Lambda(A)$ is a Borel set in $X$.

Proof. Let $N$ be the counting function just introduced and define the stopping time

$$
n(x):=\min \{n \in \mathbb{N}: x \text { belongs to the closure of } N(x) \text { qubes at level } n\} .
$$


We need the sets

$$
H_{k, n}=\{x: N(x)=k \text { and } n(x)=n\}
$$

and their pieces

$$
H_{k, n}\left(\alpha_{1}, \ldots, \alpha_{k}\right)=H_{k, n} \cap \bar{Q}_{\alpha_{1}} \cap \cdots \cap \bar{Q}_{\alpha_{k}},
$$

where $\alpha_{1}, \ldots \alpha_{k} \in \mathcal{I}_{n}$ (index set for the qubes at level $n$ ) are distinct and their order in the labeling of the set $H_{k, n}\left(\alpha_{1}, \ldots, \alpha_{k}\right)$ does not matter.

We claim that each set $H_{k, n}\left(\alpha_{1}, \ldots, \alpha_{k}\right)$ is Borel measurable in $X$. Some of these sets and of the sets introduced below are empty, but this causes us no trouble. Set $E_{k}=\{x \in$ $X: N(x) \geq k\}$. Then, $E_{1}=X$ and

$$
E_{k}=\bigcup_{n} \bigcup_{\begin{array}{c}
\alpha_{1}, \ldots, \alpha_{k} \\
\text { distinct in } \mathcal{I}_{n}
\end{array}}\left(\bar{Q}_{\alpha_{1}} \cap \cdots \cap \bar{Q}_{\alpha_{k}}\right) .
$$

Each $E_{k}$ is Borel measurable in $X$. Set now $F_{k}:=E_{k} \backslash E_{k+1}=\{x \in X: N(x)=k\}$. Set further $G_{1,1}=F_{1}$, and

$$
G_{k, n}=F_{k} \cap\left[\bigcup_{\substack{\alpha_{1}, \ldots, \alpha_{k} \\ \text { distinct in } \mathcal{I}_{n}}}\left(\bar{Q}_{\alpha_{1}} \cap \cdots \cap \bar{Q}_{\alpha_{k}}\right)\right] .
$$

Then, $H_{k, n}=G_{k, n} \backslash G_{k, n-1}$ and the claimed measurability of the sets $H_{k, n}\left(\alpha_{1}, \ldots, \alpha_{k}\right)$ follows.

Set now, for $1 \leq j \leq k, \mathcal{H}_{k, n}^{j}\left(\alpha_{1}, \ldots, \alpha_{k}\right)$ to be the subset of $\partial T$ defined by

$$
\mathcal{H}_{k, n}^{j}\left(\alpha_{1}, \ldots, \alpha_{k}\right)=\Lambda^{-1}\left(H_{k, n}\left(\alpha_{1}, \ldots, \alpha_{k}\right)\right) \cap \partial S\left(\alpha_{j}\right) .
$$

We have the following properties, easy to verify.

(i) $\mathcal{H}_{k, n}^{j}\left(\alpha_{1}, \ldots, \alpha_{k}\right)$ is Borel measurable in $\partial T$;

(ii) For each $1 \leq j \leq k$ and each choice of distinct $\alpha_{1}, \ldots, \alpha_{k}$ in $\mathcal{I}_{n}, \Lambda$ maps $\mathcal{H}_{k, n}^{j}\left(\alpha_{1}, \ldots, \alpha_{k}\right)$ bijectively onto $H_{k, n}\left(\alpha_{1}, \ldots, \alpha_{k}\right)$;

(iii) The sets $\mathcal{H}_{k, n}^{j}\left(\alpha_{1}, \ldots, \alpha_{k}\right)$ are mutually disjoint.

We use now the sets just introduced to define a set family in $\partial T$. Namely, $\mathcal{G}$ is the family of the sets having the form

$$
\coprod_{\substack{k, n ; 1 \leq j \leq k \\ \alpha_{1}, \ldots, \alpha_{k} \in \mathcal{I}_{n}}}\left[\mathcal{H}_{k, n}^{j}\left(\alpha_{1}, \ldots, \alpha_{k}\right) \cap \Lambda^{-1}\left(A\left(k, n ; j ; \alpha_{1}, \ldots, \alpha_{k}\right)\right)\right]
$$

where each set $A\left(k, n ; j ; \alpha_{1}, \ldots, \alpha_{k}\right)$ is Borel measurable in $X$. Since $\Lambda$ is continuous, $\mathcal{G}$ is contained in the Borel $\sigma$-algebra of $\partial T$.

The family of sets $\mathcal{G}$ has in addition the properties listed below.

(i) $\mathcal{G}$ is a $\sigma$-algebra;

(ii) The image under $\Lambda$ of each set in $\mathcal{G}$ is measurable in $X$;

(iii) Each basic set $\partial S(\alpha)$ belongs to $\mathcal{G}$.

A consequence of (i),(iii) from the latter property list and (i) from the former property list, is that $\mathcal{G}$ is the Borel $\sigma$-algebra in $\partial T$ and that the image of a Borel set of $\partial T$ under $\Lambda$ is Borel in $X$. i.e., both $\Lambda$ and $\Lambda^{-1}$ map Borel sets to Borel sets. 
POTENTIAL THEORY ON TREES, GRAPHS AND AHLFORS-REGULAR METRIC SPACES

Statement (i) is easily verified:

$$
\begin{aligned}
\partial T \backslash\left\{\coprod_{\substack{k, n ; 1 \leq j \leq k \\
\alpha_{1}, \ldots, \alpha_{k} \in \mathcal{I}_{n}}}\left[\mathcal{H}_{k, n}^{j}\left(\alpha_{1}, \ldots, \alpha_{k}\right) \cap \Lambda^{-1}\left(A\left(k, n ; j ; \alpha_{1}, \ldots, \alpha_{k}\right)\right)\right]\right\}= \\
\coprod_{\substack{k, n ; 1 \leq j \leq k \\
\alpha_{1}, \ldots, \alpha_{k} \in \mathcal{I}_{n}}}\left[\mathcal{H}_{k, n}^{j}\left(\alpha_{1}, \ldots, \alpha_{k}\right) \cap \Lambda^{-1}\left(X \backslash A\left(k, n ; j ; \alpha_{1}, \ldots, \alpha_{k}\right)\right)\right]
\end{aligned}
$$

and

$$
\begin{array}{r}
\bigcup_{\lambda}\left\{\coprod_{\substack{k, n ; 1 \leq j \leq k \\
\alpha_{1}, \ldots, \alpha_{k} \in \mathcal{I}_{n}}}\left[\mathcal{H}_{k, n}^{j}\left(\alpha_{1}, \ldots, \alpha_{k}\right) \cap \Lambda^{-1}\left(A_{\lambda}\left(k, n ; j ; \alpha_{1}, \ldots, \alpha_{k}\right)\right)\right]\right\} \\
\coprod_{\substack{k, n ; 1 \leq j \leq k \\
\alpha_{1}, \ldots, \alpha_{k} \in \mathcal{I}_{n}}}\left[\mathcal{H}_{k, n}^{j}\left(\alpha_{1}, \ldots, \alpha_{k}\right) \cap \Lambda^{-1}\left(\bigcup_{\lambda} A\left(k, n ; j ; \alpha_{1}, \ldots, \alpha_{k}\right)\right)\right]
\end{array}
$$

For statement (ii), observe that

$$
\begin{array}{r}
\Lambda\left(\mathcal{H}_{k, n}^{j}\left(\alpha_{1}, \ldots, \alpha_{k}\right) \cap \Lambda^{-1}\left(A\left(k, n ; j ; \alpha_{1}, \ldots, \alpha_{k}\right)\right)\right)= \\
H_{k, n}\left(\alpha_{1}, \ldots, \alpha_{k}\right) \cap A\left(k, n ; j ; \alpha_{1}, \ldots, \alpha_{k}\right),
\end{array}
$$

which is measurable in $X$.

We now consider (iii). For fixed $\alpha$ in $T$, we want to show that $\partial S(\alpha)$ is an element of $\mathcal{G}$. Consider the sets

$$
A=\coprod_{\beta \geq \alpha} \coprod_{k=d(\beta)} \coprod_{\alpha_{1}, \ldots, \alpha_{k}: \alpha_{1}=\beta} \mathcal{H}_{k, n}^{1}\left(\alpha_{1}, \ldots, \alpha_{k}\right)
$$

and

$$
B=\coprod_{o \leq \gamma \leq \alpha} \coprod_{k, n=d(\gamma)} \coprod_{\alpha_{1}, \ldots, \alpha_{k}: \alpha_{1}=\gamma}\left(\mathcal{H}_{k, n}^{1}\left(\alpha_{1}, \ldots, \alpha_{k}\right) \cap \Lambda^{-1}\left(\overline{Q_{\alpha}}\right)\right) .
$$

By definition, $A, B \in \mathcal{G}$. It is clear that $A \subseteq \partial S(\alpha)$. We show now that $B \subseteq \partial S(\alpha)$. Let

$$
\zeta \in \mathcal{H}_{k, n}^{1}\left(\alpha_{1}, \ldots, \alpha_{k}\right) \cap \Lambda^{-1}\left(\overline{Q_{\alpha}}\right),
$$

with $n=d(\gamma)$ and let $x=\Lambda(\zeta)$. Then: $x$ has $k$ preimages at level $n$ and $\zeta$ is the the only preimage in $\partial S(\gamma)$. Suppose that $\zeta \notin \partial S(\alpha)$. Since $x \in \bar{Q}_{\alpha}$, there is some other preimage $\zeta^{\prime} \neq \zeta$ of $x$ which lies in $\partial S(\alpha)$. But $\partial S(\alpha) \subseteq \partial S(\gamma)$, hence, there are two preimages of $x$ in $\partial S(\gamma)$, a contradiction.

We now show that $\partial S(\alpha) \subseteq A \cup B$. Let $\zeta \in \partial S(\alpha), \Lambda(\zeta)=x, N(x)=k$ and $n(x)=n$. Consider two cases.

(a) First suppose $n(x) \geq d(\alpha)$. Then there are $k$ distinct sets $\bar{Q}_{\alpha_{1}}, \ldots, \bar{Q}_{\alpha_{k}}$ at level $n$ such that $x \in H_{k, n}\left(\alpha_{1}, \ldots, \alpha_{k}\right)$ and $\zeta \in \mathcal{H}_{k, n}^{j}\left(\alpha_{1}, \ldots, \alpha_{k}\right)$ for some $1 \leq j \leq k$. It has to be $\alpha_{j} \geq \alpha$, because otherwise $\alpha$ and $\alpha_{j}$ are not order related, hence $\partial S(\alpha)$ and $\partial S\left(\alpha_{j}\right)$ are disjoint and they can not both contain $\zeta$.

(b) Suppose $n(x)<d(\alpha)$. Again, $x \in H_{k, n}\left(\alpha_{1}, \ldots, \alpha_{k}\right)$ and $\zeta \in \mathcal{H}_{k, n}^{j}\left(\alpha_{1}, \ldots, \alpha_{k}\right)$ for some $1 \leq j \leq k$. We want to prove that $\gamma:=\alpha_{j}<\alpha$. If such is not the case, then $S\left(\alpha_{j}\right)$ and $S(\alpha)$ are not order-related, as above, hence they are disjoint. 
We list the basic properties of $\Lambda^{*}$.

Proposition 16. The measure $\Lambda^{*}(\omega)$ is a Borel measure on $\partial T$. Moreover,

(i) $\Lambda_{*}\left(\Lambda^{*}(\omega)\right)=\omega$;

(ii) Let $N=\max \{N(x): x \in X\}$. Then, for each Borel measurable set $A$ in $\partial T$,

$$
\frac{\omega(\Lambda(A))}{N} \leq \Lambda^{*}(\omega)(A) \leq \omega(\Lambda(A))
$$

About the supports, we have:

(iii) $\operatorname{supp}\left(\Lambda^{*} \omega\right) \subseteq \Lambda^{-1}(\operatorname{supp}(\omega))$;

(iv) $\operatorname{supp}\left(\Lambda_{*} \nu\right)=\Lambda(\operatorname{supp}(\nu))$.

(v) $\operatorname{supp}(\omega) \subseteq \Lambda\left(\operatorname{supp}\left(\Lambda^{*} \omega\right)\right)$.

A consequence of $(\mathrm{v})$ is that $\Lambda^{-1}(\operatorname{supp}(\omega)) \subseteq \Lambda^{-1}\left(\Lambda\left(\operatorname{supp}\left(\Lambda^{*} \omega\right)\right)\right)$. We believe that more is true:

Conjecture 17. $\Lambda^{-1}(\operatorname{supp}(\omega)) \subseteq \operatorname{supp}\left(\Lambda^{*} \omega\right)$ (hence, by (iii), $\left.\Lambda^{-1}(\operatorname{supp}(\omega))=\operatorname{supp}\left(\Lambda^{*} \omega\right)\right)$.

Unfortunately we do not have a proof for this.

Proof of Proposition 16. First, we show that the function $N_{A}$ is measurable, so that the integral defining $\Lambda^{*}(\omega)$ makes sense. By Lemma 15, the set $A$ can be decomposed as the disjoint, countable union of measurable subsets $A_{i}$ of $\partial T$, with the property that $\Lambda$ is $1-1$ on each of them and $\Lambda\left(A_{i}\right)$ is measurable in $X$. Then,

$$
N_{A}=\sum_{i} N_{A_{i}}=\sum_{i} \chi_{\Lambda\left(A_{i}\right)}
$$

which is Borel measurable.

The set function $\Lambda^{*}(\omega)$ is additive. Let $\left\{A_{n}\right\}_{n=0}^{\infty}$ be a disjoint family of measurable sets in $\partial T$ :

$$
\begin{aligned}
\int_{X} \frac{N_{\cup A_{n}}(x)}{N(x)} d \omega(x) & =\int_{X} \frac{\sharp\left\{\zeta \in \cup A_{n}: \Lambda(\zeta)=x\right\}}{N(x)} d \omega(x) \\
& =\sum_{n} \int_{X} \frac{\sharp\left\{\zeta \in A_{n}: \Lambda(\zeta)=x\right\}}{N(x)} d \omega(x) \\
& =\sum_{n} \int_{X} \frac{N_{A_{n}}(x)}{N(x)} d \omega(x)
\end{aligned}
$$

Estimate (ii) follows from Lemmas 13 and 14. Given a measurable set $A$ in $\partial T$ and $x$ in $\Lambda(A): 1 \leq N_{A}(x) \leq N(x) \leq c$. To show (i), let $E$ be a measurable subset of $X$ :

$$
\begin{aligned}
\Lambda_{*}\left(\Lambda^{*}(\omega)\right)(E) & =\Lambda^{*}(\omega)\left(\Lambda^{-1}(E)\right)=\int_{X} \frac{N_{\Lambda^{-1}(E)}(x)}{N(x)} d \omega(x) \\
& =\int_{X} \frac{\sharp\left\{\zeta \in \Lambda^{-1}(E): \Lambda(\zeta)=x\right\}}{N(x)} d \omega(x) \\
& =\int_{X} \chi_{E}(x) \frac{N(x)}{N(x)} d \omega(x)=\omega(E) .
\end{aligned}
$$

We prove $(\mathrm{v})$. Let $K=\operatorname{supp}\left(\Lambda^{*} \omega\right)$. Then, 


$$
0=\Lambda^{*} \omega(\partial T \backslash K)=\int_{X} \frac{N_{\partial T \backslash K}(x)}{N(x)} d \omega(x)
$$

if and only if $\omega$-a.e. $(x)$ we have $0=N_{\partial T \backslash K}(x)=\sharp\left(\Lambda^{-1}(x) \cap[\partial T \backslash K]\right)$, which is equivalent to having $\Lambda^{-1}(x) \subseteq K$ for $\omega-$ a.e. $x$; hence, $x \in \Lambda(K)$ for $\omega-$ a.e. $x$. Since $\Lambda(K)$ is closed in $X, \operatorname{supp}(\omega) \subseteq \Lambda(K)=\Lambda\left(\operatorname{supp}\left(\Lambda^{*} \omega\right)\right)$.

We prove (iii). Let $E=\operatorname{supp}(\omega)$. If $x \in E, \Lambda^{-1}(x) \subseteq \Lambda^{-1}(E)$, hence $\Lambda^{-1}(x) \cap[\partial T \backslash$ $\left.\Lambda^{-1}(E)\right]=\emptyset$. Thus,

$$
\begin{aligned}
\Lambda^{*} \omega\left(\partial T \backslash \Lambda^{-1}(E)\right) & =\int_{X} \frac{\sharp\left(\Lambda^{-1}(x) \cap\left[\partial T \backslash \Lambda^{-1}(E)\right]\right)}{\sharp\left(\Lambda^{-1}(x)\right)} d \omega(x) \\
& =\int_{X \backslash E} \frac{\sharp\left(\Lambda^{-1}(x) \cap\left[\partial T \backslash \Lambda^{-1}(E)\right]\right)}{\sharp\left(\Lambda^{-1}(x)\right)} d \omega(x) \\
& =0 .
\end{aligned}
$$

i.e., $\operatorname{supp}\left(\Lambda^{*} \omega\right) \subseteq \Lambda^{-1}(E)=\Lambda^{-1}\left(\Lambda^{*} \omega\right)$.

Statement (iv) is well known and we include a proof for completeness. For $F$ closed in $\partial T$

$$
\begin{aligned}
\operatorname{supp}\left(\Lambda_{*} \omega\right) \subseteq F & \Longleftrightarrow \\
0 & =\Lambda_{*} \omega(X \backslash F)=\omega\left(\Lambda^{-1}(X \backslash F)\right) \\
& =\omega\left(\partial T \backslash \Lambda^{-1}(F)\right) \\
& \Longleftrightarrow \operatorname{supp}(\omega) \subseteq \Lambda^{-1}(F) \\
& \Longleftrightarrow \Lambda(\operatorname{supp}(\omega)) \subseteq F
\end{aligned}
$$

Remark 18. For the measure $m$ itself, we have that $N_{A}(x) / N(x)=1, m$-a.e. For each $\alpha \in$ $T$ we have $m\left(Q_{\alpha}\right)=\tilde{m}(\partial S(\alpha)): m$, the measure on $X$, and $\tilde{m}$, the measure on $\partial T$, coincide on families of generators of the corresponding Borel $\sigma$-algebras. Hence, $m(A)=\tilde{m}\left(\Lambda^{-1}(A)\right)$ for all Borel sets $A$ in $X$ and $m(\Lambda(B))=\tilde{m}(B)$ for all Borel sets $B$ in $\partial T$. We might, and we will, identify $m$ and $\tilde{m}$.

We will also need a "localized" version of $\Lambda^{*}$. Let $F$ be a closed subset of $\partial T$ and $\omega$ be a Borel measure on $X$. Then,

$$
\Lambda_{F}^{*}(A):=\Lambda^{*}(F \cap A),
$$

is the restriction of $\Lambda^{*}$ to $F$. Proposition 16 localizes to:

Corollary 19. Let $\omega$ be a positive Borel measure on $X$ and $F$ a closed set in $\partial T$. Then,

(i) $\operatorname{supp}\left(\Lambda_{F}^{*} \omega\right) \subseteq \Lambda^{-1}(\operatorname{supp}(\omega)) \cap F$.

(ii) $\Lambda_{F}^{*} \omega(A) \approx \omega(\Lambda(F \cap A))$ when $A \subseteq \partial T$ is measurable.

(iii) $\Lambda_{*}\left(\Lambda_{F}^{*} \omega\right)(E) \approx \omega(E \cap \Lambda(F))$ when $E \subseteq X$ is measurable.

Proof. (i) follows from (iii) in Proposition 16. (ii) follows from (ii) in Proposition 16. (iii) follows form (i) and (ii) in Proposition 16. 


\section{The Muckenhoupt-Wheeden Inequality on Graphs}

In this section we prove the Muckenhoupt-Wheeden inequality on graphs. In the linear case, the inequality can be proved by Fubini's Theorem and easy geometric considerations. The main point of the inequality consists in inverting "on average" the $\ell^{1} \subseteq \ell^{\infty}$ inclusion. We need its corollary: an inversion "on average" of the $\ell^{1} \subseteq \ell^{p^{\prime}}$ inclusion, which was independently proved by T. Wolff by means of a completely different argument. The other half, which inverts $\ell^{p^{\prime}} \subseteq \ell^{\infty}$, was independently proved in [AR]. The usefulness of the inequality will be shown in the next section.

Fix $0<s<1$ and $q \geq 1$. For each $x$ in $X$, let $P_{G}(x)=\left\{\alpha \in G: d_{G}\left(\alpha, \Lambda^{-1}(x)\right) \leq 1\right\}$, i.e., the set of those $\alpha$ in $G$ having distance at most one from a tree-geodesic ending at $x$.

Given a Borel measure $\omega$ on $X$, define

$$
\mathcal{I}_{G} \omega(x)=\sum_{\alpha \in P_{G}(x)} \frac{\omega(\alpha)}{m(\alpha)^{s}}
$$

and

$$
S_{G} \omega(x)=\sup _{\alpha \in P_{G}(x)} \frac{\omega(\alpha)}{m(\alpha)^{s}} .
$$

Here, $\omega(\alpha):=\omega\left(\overline{Q_{\alpha}^{k}}\right)$ and $m(\alpha):=m\left(\overline{Q_{\alpha}^{k}}\right)=m\left(Q_{\alpha}^{k}\right)$ are the measures of the corresponding sets.

Clearly, $S_{G} \omega \leq \mathcal{I}_{G} \omega$, pointwise. The following surprising theorem of Muckenhoupt and Wheeden shows that, on average, the opposite inequality holds, as well.

Theorem 20. Given $q \geq 1$, there is a constant $c_{7}$ such that

$$
\int_{X} \mathcal{I}_{G} \omega(x)^{q} d m(x) \leq c_{7} \int_{X} S_{G} \omega(x)^{q} d m(x) .
$$

The inequality was proved in $[\mathrm{MW}]$ for Riesz potentials. In [HW], T. Wolff independently rediscovered, with a different proof, one-half of it. In our context, Wolff's inequality reads:

$$
\int_{X} \mathcal{I}_{G} \omega(x)^{q} d m(x)=\int_{X}\left(\sum_{\alpha \in P_{G}(x)} \frac{\omega(\alpha)}{m(\alpha)^{s}}\right)^{q} d m(x) \leq C \int_{X} \sum_{\alpha \in P_{G}(x)} \frac{\omega(\alpha)^{q}}{m(\alpha)^{s q}} d m(x) .
$$

The left hand side is related with a classical capacity on $X$, the right hand side with a new capacity on the tree, as we will see below. The equivalence of the two definitions was the key to extend to the nonlinear case important features of linear potential theory [HW]. The other half of (10),

$$
\int_{X} \sum_{\alpha \in P_{G}(x)} \frac{\omega(\alpha)^{q}}{m(\alpha)^{s q}} d m(x) \leq C \int_{X} S_{G} \omega(x)^{q} d m(x)
$$

was later rediscovered in $[\mathrm{AR}]$, with a completely different proof.

For ease of the reader, we give the proof of Theorem 20 in the present context. First, define the maximal function

$$
M_{G}^{m} \omega(x)=\sup _{\alpha \in P_{G}(x)} \frac{\omega(\alpha)}{m(\alpha)} .
$$


POTENTIAL THEORY ON TREES, GRAPHS AND AHLFORS-REGULAR METRIC SPACES

We then have the following well-known lemma whose proof is a standard argument.

Lemma 21. The maximal function $M_{G}^{m}$ is bounded on $L^{\infty}$ and on $L^{1, \infty}$,

$$
m\left(\left\{M_{G}^{m} \omega>\lambda\right\}\right) \lambda \leq c\|\omega\|_{1}
$$

Proof of Theorem 20. Again, we separate the proof into some easier steps.

Step 1. Fix $k \in \mathbb{N}$. Let $\|\omega\|_{1}=\int_{X} d \omega$ and using Ahlfors-regularity,

$$
\begin{aligned}
\mathcal{I}_{G} \omega(x) & =\sum_{\substack{\alpha \in P_{G}(x), d(\alpha)<k \\
m(\alpha)^{s}}} \frac{\omega(\alpha)}{\sum_{\alpha \in P_{G}(x), d(\alpha) \geq k}} \frac{\omega(\alpha)}{m(\alpha)^{s}} \\
& \leq c\|\omega\|_{1} \delta^{-Q s k}+c M_{G}^{m} \omega(x) \delta^{Q(1-s) k} .
\end{aligned}
$$

Choose $k$ in such a way $\delta^{Q k} \approx\|\omega\|_{1} / M_{G}^{m} \omega(x)$, so that the two summands are approximatively equal,

$$
\|\omega\|_{1} \delta^{-Q s k} \approx M_{G}^{m} \omega(x) \delta^{Q(1-s) k}
$$

Then,

$$
\mathcal{I}_{G} \omega(x) \leq c\|\omega\|_{1}^{1-s}\left(M_{G}^{m} \omega(x)\right)^{s} .
$$

Using Lemma 21 and the estimates for $\mathcal{I}_{G}$, we obtain

$$
\begin{aligned}
m\left(\mathcal{I}_{G} \omega>\lambda\right) & \leq m\left(c\|\omega\|_{1}^{1-s}\left(M_{G}^{m} \omega(x)\right)^{s}>\lambda\right) \\
& \leq c\|\omega\|_{1} \lambda^{-\frac{1}{s}}\|\omega\|_{1}^{\frac{1-s}{s}}=c\left(\frac{\|\omega\|_{1}}{\lambda}\right)^{1 / s} .
\end{aligned}
$$

Step 2. We will show that there exist $a>1, b>1$ such that, for all $\epsilon \in(0,1)$ and all $\lambda>0$,

$$
m\left(\mathcal{I}_{G} \omega>a \lambda\right) \leq b \epsilon^{q} m\left(\mathcal{I}_{G} \omega>\lambda\right)+m\left(S_{G} \omega>\epsilon \lambda\right) .
$$

By Corollary 3, we can replace each of the three sets $A$ whose $m$-measure is considered in (15) by $A \backslash F$, considering only those points $x$ in $X$ such that $\sharp \Lambda^{-1}(x)=1$.

First, we extend the definitions of $\mathcal{I}_{G} \omega$ and $S_{G} \omega$ to points $\alpha$ of $T \equiv G$. Let $P_{G}(\alpha)=\{\beta \in$ $G: d_{G}(\beta,[o, \alpha]) \leq 1$ and $\left.d(\beta) \leq d(\alpha)\right\}$ and set

$$
\mathcal{I}_{G} \omega(\alpha):=\sum_{\beta \in P_{G}(\alpha)} \frac{\omega(\beta)}{m(\beta)^{s}},
$$

and

$$
S_{G} \omega(\alpha):=\sup _{\beta \in P_{G}(\alpha)} \frac{\omega(\beta)}{m(\beta)^{s}} .
$$

If $\mathcal{I}_{G} \omega(x)>\lambda$ and $x \notin F$, then there is $\alpha \in P_{G}(x)$ such that $\mathcal{I}_{G} \omega(\alpha)>\lambda$ and it is clear that, if $\alpha>\beta$ in $T$, then $\mathcal{I}_{G} \omega(\alpha) \geq \mathcal{I}_{G} \omega(\beta)$. Let $A(\lambda)$ be the set of the $\alpha$ in $T$ which are maximal with the property $\mathcal{I}_{G} \omega(\alpha)>\lambda$.

Then, the set $\left\{\xi \in(X \backslash F) \cup G: \mathcal{I}_{G} \omega(\xi)>\lambda\right\}$ is the disjoint union of the sets $S_{1}(\alpha)=$ $\left.S(\alpha) \cup \overline{\left(Q_{\alpha}^{d(\alpha)}\right.} \backslash F\right)$, as $\alpha$ ranges in $A(\lambda)$.

Observe that $\partial_{\bar{G}} S(\alpha)=\overline{Q_{\alpha}^{d(\alpha)}}$ is the boundary of $S(\alpha)$ with respect to the metric $\bar{\rho}$ in $\bar{G}$.

By maximality, if $\alpha$ is in $A(\lambda)$, then $\mathcal{I}_{G} \omega\left(\alpha^{-1}\right) \leq \lambda<\mathcal{I}_{G} \omega(\alpha)$. 
Fix $\alpha$ in $A(\lambda)$. We consider two cases. Suppose that for $m$-almost all $x$ in $\overline{Q_{\alpha}^{d(\alpha)}}$ we have $S_{G} \omega(x)>\epsilon \lambda$. Then,

$$
m\left(\left\{\mathcal{I}_{G} \omega>a \lambda\right\} \cap \partial_{\bar{G}} S(\alpha)\right) \leq m\left(\partial_{\bar{G}} S(\alpha)\right)=m\left(\left\{S_{G} \omega>\epsilon \lambda\right\} \cap \partial_{\bar{G}} S(\alpha)\right),
$$

and we have nothing to prove.

Suppose that, on the contrary, there is $x_{0}$ in $\overline{Q_{\alpha}} \backslash F$ such that $S_{G} \omega\left(x_{0}\right) \leq \epsilon \lambda$. Let

$$
\tilde{S}(\alpha)=\bigcup_{\substack{\beta: d(\beta)=d(\alpha) \\ \text { a } \\ d_{G}(\alpha, \beta) \leq 1}} \overline{Q_{\beta}},
$$

and let $\omega_{1}=\left.\omega\right|_{\tilde{S}(\alpha)}$.

We write $\partial_{\bar{\rho}} \tilde{S}(\alpha)=\cup_{\beta} \partial_{\bar{\rho}} S(\beta)$, the union being over the $\beta$ 's used in the definition of $\tilde{S}(\alpha)$.By Step 1 ,

$$
m\left(\left\{\mathcal{I}_{G} \omega_{1}>\frac{a \lambda}{2}\right\} \cap \overline{Q_{\alpha}}\right) \leq \frac{c}{a^{1 / s}}\left(\frac{\left\|\omega_{1}\right\|_{1}}{\lambda}\right)^{1 / s}
$$

We estimate

$$
\left\|\omega_{1}\right\|_{1}=\omega\left(\partial_{\bar{\rho}} \tilde{S}(\alpha)\right) \leq c S_{G} \omega(\alpha) m(\alpha)^{s}
$$

In fact,

$$
\begin{aligned}
& \omega\left(\partial_{\bar{\rho}} \tilde{S}(\alpha)\right) \leq \sum_{\substack{\beta: d(\beta)=d(\alpha) \\
d_{G}(\alpha, \beta) \geq 1}} \omega\left(\partial_{\bar{\rho}} S(\beta)\right) \\
& \leq \quad c \max _{\substack{\beta: d(\beta)=d(\alpha) \\
d_{G}(\alpha, \beta) \geq 1}} \omega\left(\partial_{\bar{\rho}} S(\beta)\right) \\
& \text { because there are boundedly many such } \beta \text { 's } \\
& \leq c\left(\max _{\substack{\beta: d(\beta)=d(\alpha) \\
d_{G}(\alpha, \beta) \geq 1}} \frac{\omega(\beta)}{m(\beta)^{s}}\right) m(\alpha)^{s} \\
& \leq c S_{G} \omega(\alpha) m(\alpha)^{s} \text {. }
\end{aligned}
$$

Inserting (17) in (16),

$$
\begin{aligned}
m\left(\left\{\mathcal{I}_{G} \omega_{1}>\frac{a \lambda}{2}\right\} \cap \alpha\right) & \leq c \frac{m(\alpha)}{a^{1 / s}}\left(\frac{S_{G} \omega(\alpha)}{\lambda}\right)^{1 / s} \\
\leq & \frac{c}{a^{1 / s}} m(\alpha) \epsilon^{1 / s} \\
& \text { since } S_{G} \omega(\alpha) \leq S_{G} \omega\left(x_{0}\right) \leq \epsilon \lambda \\
\leq & c m(\alpha) \epsilon^{1 / s} .
\end{aligned}
$$

Consider now $\omega_{2}:=\omega-\omega_{1}$. Then,

$$
\mathcal{I}_{G} \omega_{2}\left(\alpha^{-1}\right) \leq \mathcal{I}_{G} \omega\left(\alpha^{-1}\right) \leq \lambda,
$$

by the maximality of $\alpha$. If $x \in \alpha \backslash F$, then,

$$
\mathcal{I}_{G} \omega_{2}(x)=\mathcal{I}_{G} \omega_{2}\left(\alpha^{-1}\right) \leq \lambda<\frac{a \lambda}{2} .
$$

In the first equality we use the information on $\operatorname{supp}\left(\omega_{2}\right)$. 
Thus,

$$
\begin{aligned}
\left\{\mathcal{I}_{G} \omega>a \lambda\right\} \cap(\alpha \backslash F) & =\left[\left\{\mathcal{I}_{G} \omega_{1}>\frac{a \lambda}{2}\right\} \cap(\alpha \backslash F)\right] \cup\left[\left\{\mathcal{I}_{G} \omega_{2}>\frac{a \lambda}{2}\right\} \cap(\alpha \backslash F)\right] \\
& =\left[\left\{\mathcal{I}_{G} \omega_{1}>\frac{a \lambda}{2}\right\} \cap(\alpha \backslash F)\right] .
\end{aligned}
$$

The wished estimate follows from (18) and (19), proving Step 2.

Step 3. A "standard argument" (see $[\mathrm{AH}]$ ) shows that the estimate follows from the good- $\lambda$ inequality in Step 2.

For our purposes, the main point of interest of Theorem 20 is the following.

\section{Corollary 22.}

$$
\int_{X}\left(\sum_{\alpha \in P_{G}(x)} \frac{\omega(\alpha)}{m(\alpha)^{s}}\right)^{q} d m(x) \leq c \int_{X} \sum_{\alpha \in P_{G}(x)}\left(\frac{\omega(\alpha)}{m(\alpha)^{s}}\right)^{q} d m(x) .
$$

\section{Proof of Theorem 1}

In this section, we first write the energy $\mathcal{E}(\omega)$ of a measure $\omega$ on $X$ in a way which resembles one side of the Muckenhoupt-Wheeden inequality, the use Wolff's half of the inequality to approximatively write the energy in a different way. The catch is that when the same procedure is applied to a measure on $\partial T$, one gets a very similar expression: the approximating expression for the energy, in fact, does not depend on the graph geometry, but only on the tree geometry. Using the fact that Borel measures on $\partial T$ and on $X$ can be moved forth and back through $\Lambda$, and observing that energies of corresponding measures are equivalent, we easily obtain a proof of the equivalence of capacities in Theorem 1.

Lemma 23. There are positive constants $c^{\prime}, c^{\prime \prime}$ independent of the Borel measure $\omega$ and of the point $x$ in $X$ such that

$$
c^{\prime} \sum_{\alpha \in P_{G}(x),} m(\alpha)^{-s} \leq K(x, y) \leq c^{\prime \prime} \sum_{\alpha \in P_{G}(x), y \in \alpha} m(\alpha)^{-s}
$$

and

$$
c \mathcal{I}_{G} \omega(x) \leq K \omega(x) \leq c \mathcal{I}_{G} \omega(x) .
$$

Proof. We begin with the estimate from below.

$$
\begin{aligned}
\mathcal{I}_{G} \omega(x) & =\sum_{\alpha \in P_{G}(x)} \frac{\omega(\alpha)}{m(\alpha)^{s}} \\
& =\int_{X}\left(\sum_{\alpha \in P_{G}(x)} \frac{\chi_{\alpha}(y)}{m(\alpha)^{s}}\right) d \omega(y) \\
& =\int_{X}\left(\sum_{\alpha \in P_{G}(x) \cap P_{G}^{0}(y)} m(\alpha)^{-s}\right) d \omega(y),
\end{aligned}
$$

where $P_{G}^{0}(y)$ is the union of the tree geodesics ending at $y$. Let $\alpha$ be one of the indexes over which the sum is taken. Then, $y \in \alpha$ and there is $\beta_{G}^{\sim} \alpha$ such that $x \in \beta$. Then, $\bar{\rho}(x, y) \leq c \delta^{d(\alpha)}$. Also, at each level $k$, there are boundedly many $\alpha$ indexing the sum. Thus, 


$$
\begin{aligned}
\left(\sum_{\alpha \in P_{G}(x) \cap P_{G}^{0}(y)} m(\alpha)^{-s}\right) & \approx \sum_{\substack{\alpha: x \in \alpha, \bar{\rho}(x, y) \leq \delta d(\alpha)\\
}} m(\alpha)^{-s} \\
& \approx m(x, \bar{\rho}(x, y))^{-s} \approx K(x, y) .
\end{aligned}
$$

This is the first estimate in the lemma; the second follows by inserting (21) in (20).

Given the kernel $K$ on $X$, we define a new kernel $K_{\partial T}$ on $\partial T$, with $\tilde{m}$ instead of $m$ and with $\rho_{T}$ instead of $\rho \approx \bar{\rho}$.

Theorem 24. Given a measure $\omega$ in $X$, let $\Lambda^{*}(\omega)$ be the measure on $\partial T$ defined by (9), and let $\mathcal{E}_{\partial T}$ be the energy defined by the kernel $K_{\partial T}$. Then,

$$
\mathcal{E}_{X}(\omega) \approx \mathcal{E}_{\partial T}\left(\Lambda^{*} \omega\right) .
$$

On the other hand, if $\nu$ is a measure on $\partial T$, then

$$
\mathcal{E}_{\partial T}(\nu) \approx \mathcal{E}_{X}\left(\Lambda_{*} \nu\right)
$$

\section{Proof.}

We first prove (22)

$$
\begin{aligned}
\mathcal{E}_{X}(\omega) & :=\int_{X} K \omega^{p^{\prime}} d m \\
& \approx \int_{X}\left[\mathcal{I}_{G} \omega\right]^{p^{\prime}} d m \text { by Lemma } 23 \\
& \approx \int_{X} \sum_{\alpha \in P_{G}(x)}\left(\frac{\omega(\alpha)}{m(\alpha)^{s}}\right)^{p^{\prime}} d m(x) \text { by Theorem B } \\
& =\sum_{\alpha \in T} \frac{\omega(\alpha)^{p^{\prime}}}{m(\alpha)^{s p^{\prime}}} \int_{X} \chi\left(x \in X: \alpha \in P_{G}(x)\right) d m(x) \\
& \approx \sum_{\alpha \in T} \frac{\omega(\alpha)^{p^{\prime}}}{m(\alpha)^{s p^{\prime}-1}} \\
& \approx \sum_{\alpha \in T} \frac{\Lambda^{*}(\omega)(\alpha)^{p^{\prime}}}{\tilde{m}(\alpha)^{s p^{\prime}-1}},
\end{aligned}
$$

by Proposition 16 .

The key observation is that the last expression in the chain of equivalences does not depend on the graph structure, but only on the structure of $T$. In fact, particularizing the calculations of (24) to the case $X=\partial T, G=T$, and to the measure $\mu=\Lambda^{*}(\omega)$ instead of $\omega$, we obtain

$$
\mathcal{E}_{\partial T}\left(\Lambda^{*}(\omega)\right) \approx \sum_{\alpha \in T} \frac{\Lambda^{*}(\omega)(\alpha)^{p^{\prime}}}{\tilde{m}(\alpha)^{s p^{\prime}-1}} .
$$

We have then the desired estimate $\mathcal{E}_{\partial T}\left(\Lambda^{*}(\omega)\right) \approx \mathcal{E}_{X}(\omega)$.

We now consider (23). Recall the identification $\alpha \equiv \overline{Q_{\alpha}}$ between points in $G$ and the closure of dyadic sets in $X$. In the tree $T, \alpha$ is identified with a dyadic subregion $[\alpha] \subseteq \partial T$ in the same fashion, and $[\alpha] \subset \Lambda^{-1}(\alpha)$. The inclusion is generally proper because of the "edge 
effect": elements in the boundary of $\overline{Q_{\alpha}}$ have preimages in dyadic subsets on $\partial T$ at the same level of $[\alpha]$, but different from $[\alpha]$.

However, it is still true that $\Lambda^{-1}(\alpha) \subset \cup_{\beta_{G}{ }^{\alpha}}[\beta]$, and this, together with Ahlfors-regularity and the uniform bound on the number of such $\beta$ 's, suffices to show that (using the identification of the measures $m$ on $X$ with the measure $\Lambda^{*} m$ on $\partial T$, see in Remark 18):

$$
\begin{aligned}
\mathcal{E}_{\partial T}(\nu) & \approx \sum_{\alpha} \frac{\nu([\alpha])^{p^{\prime}}}{m(\alpha)^{s p^{\prime}-1}} \\
& \approx \sum_{\beta} \frac{\Lambda_{*} \nu(\beta)^{p^{\prime}}}{m(\beta)^{s p^{\prime}-1}} \\
& \approx \mathcal{E}_{X}\left(\Lambda_{*} \nu\right) .
\end{aligned}
$$

We are now ready for the proof of Theorem 1.

Proof of Theorem 1. Observe that the first assertion (4) is implied by the second, since (5) implies that

$$
\operatorname{cap}_{\partial T}\left(\Lambda^{-1}\right)(E) \approx \operatorname{cap}_{X}\left(\Lambda\left(\Lambda^{-1}\right)(E)\right)=\operatorname{cap}_{X}(E) .
$$

Let $F \subseteq \partial T$ be closed. We first show that

$$
\operatorname{cap}_{X}(\Lambda(F)) \lesssim \operatorname{cap}_{\partial T}(F)
$$

Let $\omega$ be a positive Borel measure on $X$, supported on $\Lambda(F)$. Then, $\operatorname{supp}\left(\Lambda_{F}^{*} \omega\right) \subseteq F$. Also,

$$
\begin{aligned}
\left\|\Lambda_{F}^{*} \omega\right\|_{1} & =\Lambda^{*} \omega(F) \\
& =\int_{X} \frac{\sharp\left(\Lambda^{-1}(x) \cap F\right)}{\sharp\left(\Lambda^{-1}(x)\right)} d \omega(x) \\
& \approx \omega(\Lambda(F))=\|\omega\|_{1} .
\end{aligned}
$$

About energies, since we restrict the sets to $F$, the estimate in (22) is one-side only:

$$
\begin{aligned}
\mathcal{E}_{X}(\omega) & \approx \sum_{\alpha \in T} \frac{\omega\left(\overline{Q_{\alpha}}\right)^{p^{\prime}}}{m\left(Q_{\alpha}\right)^{p^{\prime} s-1}} \\
& \approx \sum_{\alpha \in T} \frac{\Lambda^{*} \omega(\partial S(\alpha))^{p^{\prime}}}{m\left(Q_{\alpha}\right)^{p^{\prime} s-1}} \\
& \gtrsim \sum_{\alpha \in T} \frac{\Lambda_{F}^{*} \omega(\partial S(\alpha))^{p^{\prime}}}{m\left(Q_{\alpha}\right)^{p^{\prime} s-1}} \\
& \approx \mathcal{E}_{\partial T}\left(\Lambda_{F}^{*} \omega\right) .
\end{aligned}
$$

These three information together give:

$$
\begin{aligned}
\operatorname{cap}_{X}(\Lambda(F)) & =\sup \left\{\frac{\|\omega\|_{1}^{p}}{\mathcal{E}_{X}(\omega)^{p-1}}: \operatorname{supp}(\omega) \subseteq \Lambda(F)\right\} \\
& \lesssim \sup \left\{\frac{\left\|\Lambda_{F}^{*}(\omega)\right\|_{1}^{p}}{\mathcal{E}_{\partial T}\left(\Lambda_{F}^{*} \omega\right)^{p-1}}: \operatorname{supp}\left(\Lambda_{F}^{*}(\omega)\right) \subseteq F\right\} \\
& \leq \operatorname{cap}_{\partial T}(F) .
\end{aligned}
$$


In the other direction, we show that, if $F$ is closed in $\partial T$, then

$$
\operatorname{cap}_{X}(\Lambda(F)) \gtrsim \operatorname{cap}_{\partial T}(F)
$$

Let $\nu$ be a positive Borel measure supported on $F$. Then, $\operatorname{supp}\left(\Lambda_{*} \nu\right)=\Lambda(\operatorname{supp}(\nu)) \subseteq \Lambda(F)$, by Lemma 16 (iv). Hence,

$$
\begin{aligned}
\operatorname{cap}_{\partial T}(F) & =\sup \left\{\frac{\|\nu\|_{1}^{p}}{\mathcal{E}_{\partial T}(\nu)^{p-1}}: \operatorname{supp}(\nu) \subseteq F\right\} \\
& \lesssim \sup \left\{\frac{\left\|\Lambda_{*} \nu\right\|_{1}^{p}}{\mathcal{E}_{X}\left(\Lambda_{*} \nu\right)^{p-1}}: \operatorname{supp}\left(\Lambda_{*} \nu\right) \subseteq \Lambda(F)\right\} \\
& \leq \operatorname{Cap}_{X}(\Lambda(F))
\end{aligned}
$$

\section{Potential Theory on Trees}

In this section, we develop the basic potential theory on trees. Most material is covered, with different degrees of generality, in various sources ([So] and [LyPe], for instance).

We give a self-contained exposition, only requiring the basic facts of nonlinear potential theory, for which we refer to $[\mathrm{AH}]$ (Sect. 2.3-2.5). The reader will find these facts translated into tree language in the Appendix. We list the main features of the section.

(i) In 5.1, we identify the capacity $\mathrm{Cap}_{\partial T}$ on $\partial T$ considered before with (the restriction to $\partial T$ of) a new tree capacity Cap defined on $\bar{T}$. This is analogous to the identification, for subsets of the unit circle, of logarithmic capacity in the complex plane with the linear 1/2-Bessel capacity on circle. In the Ahlfors-regular case, as a consequence, we have the identification of Cap $_{X}$ with the new tree capacity Cap.

(ii) In 5.2, we estimate the capacity Cap of points and sets having the form $\partial S(\alpha)(\alpha \in$ T). As a corollary, we have estimates for the capacity of metric balls in Ahlforsregular spaces. It is here explained why the cases $s \in\left[1 / p^{\prime}, 1\right)$ are the only interesting ones in the Ahlfors-regular case and why $s=1 / p^{\prime}$ is especially interesting.

(iii) We prove in Section 5.3 a recursive formula to compute capacities of closed subsets of $\bar{T}$. Indeed, the formula gives two-sided estimates for capacities in Ahlfors-regular metric spaces.

(iv) We give a simple proof, in Section 5.4, of the Trace Theorem (or Carleson Measure Theorem) on trees. The measures satisfying the Trace Inequality (or Carleson imbedding, in the language of most complex analysts) will be characterized by a testing condition, in the spirit of $[\mathrm{KS}]$.

By the general machinery developed earlier, the Trace Theorem on trees implies an analogous theorem in Ahlfors-regular spaces.

(v) In 5.5, we show that the capacity of a set in the tree can be defined by means of the "Carleson measures" supported on this set.

(vi) In 5.6, we give a direct proof-using (v) and a monotonicity property- that the testing condition of the Trace Theorem is equivalent to a certain capacitary condition. An indirect proof of this fact is that the capacitary condition, as well, characterizes the Carleson measures. 
5.1. Tree Capacity Seen from "Inside" the Tree. Let $T$ be a tree, having (combinatorial) boundary $\partial T$ with respect to a fixed root $o$ in $T$, and let $\bar{T}=T \cup \partial T$ be the compactification of $T$. The tree $T$ is endowed with a positive weight $\pi, \pi(x)>0 \forall x \in T$. We consider exponents $1<p<\infty$ and $p^{-1}+p^{\prime-1}=1$.

Let $\xi$ in $\bar{T}$. The predecessor set of $\xi$ is $P(\xi)=\{y: o \leq y \leq \xi\} \cap T$. The successor set in $\bar{T}$ of $x \in T$ is $S(x)=\{\xi \in \bar{T}: \xi \geq x\}$. The natural distance in $T$ is denoted by $d$. Given $x$ and $x$ in $T$, we define

$$
d_{\pi}(x, y)=\sum_{z \in[x, y]} \pi(z)^{1-p^{\prime}}
$$

where $[x, y]$ is the unique geodesic for the distance $d$, here considered as a set of vertices, joining $x$ and $y$. The function $d_{\pi}$ might be considered as the length-distance associated with the weight $\pi^{1-p^{\prime}}$, but for the fact that $d_{\pi}(x, x)=\pi(x)^{1-p^{\prime}} \neq 0$. If we needed the full force of a distance, we might define the weight $\pi$ on the set of the edges, rather than on the set of the vertices; this is what is generally done when studying electrical networks [So]. Weights defined on vertices, however, perfectly serve our present purposes.

The Hardy operator $I$ is defined as

$$
\operatorname{If}(\xi)=\sum_{y \in P(\xi)} f(y), \xi \in \bar{T} .
$$

Its formal adjoint is

$$
I^{*} \omega(x)=\int_{S(x)} d \omega(y), x \in T
$$

where $\omega$ is a Borel measure on $\bar{T}$.

We consider the kernel $g: \bar{T} \times T \rightarrow \mathbb{R}$ defined by

$$
g(\zeta, \alpha)=\chi_{P(\zeta)}(\alpha)=\chi_{S(\alpha)}(\zeta) .
$$

Here, we think of $\left(T, \pi^{1-p^{\prime}}\right)$ as a measure space. The set $\bar{T}$ is given the length-metric structure $\pi_{T}: \bar{T} \times \bar{T} \rightarrow[0, \infty)$ which assigns to each edge $\left(\alpha, \alpha^{-1}\right)$ the length $2^{-d(\alpha, o)}$. Its restriction to the boundary $\partial T$ of the tree $T$ is $\pi_{T}(\zeta, \xi)=C \cdot 2^{-d(\zeta \wedge \xi)}$ (the metric itself will play here no rôle: we are only interested in the topology).

It is well explained in $[\mathrm{AH}]$ how to develop the potential theory associated with the kernel $g$. See also the Appendix to the present article. The potential of a function $f=\pi^{p^{\prime}-1} \varphi: T \rightarrow \mathbb{R}$ is $G f=I\left(f \pi^{1-p^{\prime}}\right)=I \varphi: \bar{T} \rightarrow \mathbb{R}$; while the (dual) potential of a positive, Borel measure $\omega$ on $\bar{T}$ is given by $\check{G} \omega=I^{*} \omega=\int_{S(y)} d \omega$.

The energy of the measure $\omega$ is

$$
\mathcal{E}(\omega)=\sum_{\alpha \in T}\left(I^{*} \omega(\alpha)\right)^{p^{\prime}} \pi(\alpha)^{1-p^{\prime}}
$$

We can finally define the capacity $\operatorname{Cap}(E)$ of a closed subset $E$ of $\bar{T}$ as

$$
\begin{aligned}
\operatorname{Cap}(E) & =\inf \left\{\|\varphi\|_{\ell^{p}(\pi)}^{p}: \varphi \geq 0, I \varphi \geq 1 \text { on } E\right\} \\
& =\sup \left\{\frac{\omega(E)^{p}}{\mathcal{E}(\omega)^{p-1}}: \operatorname{supp}(\omega) \subseteq E\right\} .
\end{aligned}
$$

The second equality is a deep result in potential theory (see the appendices), whose proof relies on a min/max principle. 
In the important case when $T$ is the tree of Christ's dyadic boxes of an Ahlfors $Q$-regular metric space $(X, \rho, m)$ (which without essential loss of generality, in view of Theorem 1 and Proposition 12, might be taken to be $\partial T$ itself), and $0<s<1$ is fixed, we consider the weight

$$
\pi_{s}(\alpha)=m\left(\bar{Q}_{\alpha}\right)^{\frac{s p^{\prime}-1}{p^{\prime}-1}}=m(\alpha)^{\frac{s p^{\prime}-1}{p^{\prime}-1}}
$$

The case $s p^{\prime}=1$ correspond to unweighted potential theory on trees, which, as we shall see, leads to "logarithmic" potentials.

As before, we have identified the measure $m$ on $X$ with the corresponding measure $\Lambda^{*} m$ on $\partial T$.

Theorem 25. Let $T$ be the tree associated with the metric measure space $(X, \rho, m), 0<s<1$ fixed and $\pi=\pi_{s}$ be the weight just defined. Let Cap the capacity on $\bar{T}$ associated with $\pi_{s}$. Then, there are constants $C_{1}<C_{2}$ such that, for all compact subsets $K$ of $\partial T$,

$$
C_{1} \operatorname{Cap}(K) \leq \operatorname{Cap}_{X}(\Lambda(K)) \leq C_{2} \operatorname{Cap}(K) .
$$

and

$$
\operatorname{Cap}_{X}(F) \approx \operatorname{Cap}\left(\Lambda^{-1}(F)\right)
$$

whenever $F$ is closed in $\partial T$.

Theorem 25 is actually implicit in the proof of (22): comparable energies give comparable capacities. We have decide to state it separately in this section because the relation between Cap and $\mathrm{Cap}_{\partial T}$, especially in the important case $s p^{\prime}=1$, is similar to the relation between logarithmic capacity (in the complex plane) and Bessel 1/2-capacity (on the real line) for subsets of the real line.

To better contextualize the capacities in Theorem 25, consider that, when $\pi=\pi_{s}$,

$$
\begin{aligned}
\mathcal{E}(\omega) & :=\sum_{\alpha \in T}\left(I^{*} \omega(\alpha)\right)^{p^{\prime}} \pi_{s}(\alpha)^{1-p^{\prime}} \\
& =\sum_{\alpha \in T} \frac{\left.I^{*} \omega(\alpha)\right)^{p^{\prime}}}{m(\alpha)^{s p^{\prime}-1}} \\
& =\int_{\partial T} I\left[\left(I^{*} \omega\right)^{p^{\prime}-1} \pi_{s}^{1-p^{\prime}}\right](\zeta) d \omega(\zeta) \\
& =\int_{\partial T} \sum_{\alpha \in T} \frac{I^{*} \omega^{p^{\prime}}(\alpha)}{m(\alpha)^{s p^{\prime}}} d m(\zeta) .
\end{aligned}
$$

Both equalities follow from straightforward applications of Fubini's Theorem.

The integrand on the third line,

$$
W(\omega)=I\left[\left(I^{*} \omega\right)^{p^{\prime}-1} \pi^{1-p^{\prime}}\right]
$$

is the Wolff potential of the measure $\omega$; which was introduced by Wolff in [HW]. The integral in the fourth line is the intermediate term in Theorem 20, with $q=p^{\prime}$. A deep analysis of the Wolff potential and of its applications can be found in [COV] and [KV].

For the remaining part of this section, we will consider general weights $\pi$ on a generic tree $T$ : proofs are not more difficult and notation is actually more transparent. We will point out, in some cases of interest, how the results specialize to the case when $T$ is the tree of the dyadic boxes introduced by Christ. 
POTENTIAL THEORY ON TREES, GRAPHS AND AHLFORS-REGULAR METRIC SPACES

5.2. Capacity of Special Sets. We begin with a general fact: points having positive capacity are points having finite "potential theoretic distance" from the root.

Proposition 26. Let $\zeta_{0}$ be a point of $\partial T$. Then,

$$
\operatorname{Cap}\left(\left\{\zeta_{0}\right\}\right)>0 \Longleftrightarrow d_{\pi}\left(o, \zeta_{0}\right)<\infty .
$$

More precisely, $\operatorname{Cap}\left(\left\{\zeta_{0}\right\}\right)=d_{\pi}\left(0, \zeta_{0}\right)^{1-p}$ whenever $\zeta_{0} \in \bar{T}$.

Proof. It suffices to compute the energy of a unit mass $\delta_{\zeta_{0}}$ concentrated at $\zeta_{0}$ :

$$
\begin{aligned}
\mathcal{E}\left(\delta_{\zeta_{0}}\right) & =\sum_{\alpha}\left(I^{*} \delta_{\zeta_{0}}\right)^{p^{\prime}}(\alpha) \pi^{1-p^{\prime}}(\alpha) \\
& =\sum_{\alpha} \pi^{1-p^{\prime}}=d_{\pi}(\alpha) .
\end{aligned}
$$

It is obvious from the definition of capacity in $\bar{T}$ that $\operatorname{Cap}(\{\alpha\}) \geq \operatorname{Cap}(S(\alpha)$ ) (if $I \varphi$ is one on $\alpha$, it can be taken to be one on $\partial S(\alpha)$ without increasing the norm of $\varphi)$. Interesting cases are those in which the opposite inequality holds as well; for instance, the Ahlfors-regular case.

Lemma 27. If $0<s<1$ and $\pi=\pi_{s}$, then $\operatorname{Cap}(\{\alpha\}) \approx \operatorname{Cap}(\partial S(\alpha))$.

Proof. We have to prove that $\operatorname{Cap}(\{\alpha\}) \lesssim \operatorname{Cap}(\partial S(\alpha))$. A simple calculation shows that

$$
\operatorname{Cap}(\{\alpha\})=d_{\pi_{s}}(\alpha) \approx\left\{\begin{array}{l}
d(\alpha)^{1-p} \text { if } s=1 / p^{\prime} \\
m(\alpha)^{p\left(s-1 / p^{\prime}\right)} \text { if } 1 / p^{\prime}<s<1
\end{array}\right.
$$

We test the definition of capacity (28) on the measure $\omega:=\left.m\right|_{\partial S(\alpha)}$, so that $\omega(\partial S(\alpha))=$ $m(\alpha)$.

We estimate the energy of $\omega$ :

$$
\begin{aligned}
\mathcal{E}(\omega) & =\sum_{\beta \in[o, \alpha)} \frac{m(\alpha)^{p^{\prime}}}{m(\beta)^{s p^{\prime}-1}}+\sum_{\gamma \in S(\alpha)} m(\gamma)^{p^{\prime}+1-s p^{\prime}} \\
& =I+I I .
\end{aligned}
$$

For the first term we have (recognizing a geometric series, by Ahlfors-regularity, when $\left.1 / p^{\prime}<s<1\right)$ :

$$
I \approx\left\{\begin{array}{l}
d(\alpha) m(\alpha)^{p^{\prime}} \text { if } s=1 / p^{\prime}, \\
m(\alpha)^{1+p^{\prime}-s p^{\prime}} \text { if } 1 / p^{\prime}<s<1 .
\end{array}\right.
$$

For the second term, using Ahlfors-regularity and the fact that $p^{\prime}(1-s)>0$,

$$
\begin{aligned}
I I & \approx \sum_{n=0}^{\infty}\left(\sum_{\gamma \in S(\alpha), d(\gamma, \alpha)=n} m(\gamma)\right) \delta^{Q(d(\alpha)+n) p^{\prime}(1-s)} \\
& =m(\alpha) \sum_{\substack{\infty=0 \\
\delta^{\prime}}}^{Q(d(\alpha)+n) p^{\prime}(1-s)} \\
& \approx m(\alpha)^{1+p^{\prime}-s p^{\prime}} .
\end{aligned}
$$

Summing $I$ and $I I$ and using the definition of capacity,

$$
\operatorname{Cap}(\partial S(\alpha)) \geq \frac{\omega(\alpha)^{p}}{\mathcal{E}(\omega)^{p-1}}
$$




$$
\begin{aligned}
& \approx\left\{\begin{array}{l}
\frac{m(\alpha)^{p}}{\left[m(\alpha)^{p^{\prime}} d(\alpha)\right]^{p-1}} \text { if } s=1 / p^{\prime}, \\
\frac{m(\alpha)^{p}}{\left[m(\alpha)^{\left(1-s p^{\prime}+p^{\prime}\right)}\right]^{p-1}} \text { if } 1 / p^{\prime}<s<1,
\end{array}\right. \\
& =\left\{\begin{array}{l}
d(\alpha)^{1-p} \text { if } s=1 / p^{\prime} \\
m(\alpha)^{p\left(s-1 / p^{\prime}\right)} \text { if } 1 / p^{\prime}<s<1 .
\end{array}\right. \\
& =\operatorname{Cap}(\{\alpha\}),
\end{aligned}
$$

as wished.

By Theorem 1, these conclusions apply to the case of the trees coming from Ahlfors-regular spaces and $0<s<1$. A simple geometric series argument, Proposition 26 and Lemma 27 imply:

Corollary 28. Let $(X, m, \rho)$ be a (bounded) Ahlfors $Q$-regular space and $0<s<1$. Then,

(i) For all points $x$ in $X, \operatorname{Cap}_{X}(\{x\})=0$ if and only if $\frac{1}{p^{\prime}} \leq s<1$;

(ii) More generally,

$$
\operatorname{Cap}_{X}(B(x, r)) \approx\left\{\begin{array}{l}
\log \frac{1}{r} \text { if } s=1 / p^{\prime} \\
r^{Q\left(s p^{\prime}-1\right)} \text { if } 1 / p^{\prime} \leq s<1 .
\end{array}\right.
$$

As earlier anticipated, the value $s=1 / p^{\prime}$ relates the capacity Cap $_{X}$ with a logarithmic capacity in the tree. Ultimately, this fact is hidden in the Wolff inequality.

5.3. An Algorithm to Compute Tree Capacities. For the material in this subsection, relevant references are also $[\mathrm{So}]$ and $[\mathrm{BP}]$. First, we show that the capacity of a set can be computed as the "derivative at infinity" of a Green function. In this case, the rôle of the point at infinity is played by the root.

Theorem 29. Let $E$ be closed in $\bar{T}$ and let $\varphi$ be the corresponding extremal function. Then,

$$
\operatorname{Cap}(E)=\varphi^{p-1}(o) \pi(o)
$$

The Green function in question is the equilibrium potential $\Phi=I \varphi$.

Proof. Let $E$ be a closed subset of $\bar{T}$ and $\sigma, \varphi=\left(I^{*} \sigma\right)^{p^{\prime}-1} \pi^{1-p^{\prime}}$ be, respectively, its capacitary measure and capacitary function. Then,

$$
\operatorname{Cap}(E)=\sigma(E)=I^{*} \sigma(o)=\pi(o) \varphi(o)^{p-1} .
$$

Let $d_{\pi}$ be the distance associated with $\pi: d_{\pi}(x)=\sum_{y \in P(x)} \pi^{1-p^{\prime}}(y)$. The capacity of a subset of $T$ can be computed by means of a recursive algorithm.

Theorem 30. Let $Z$ be a set in $\bar{T}, x_{0}$ a point in $T$ and let $x_{1}, \ldots, x_{n}$ be its children. For each $j$, let $Z_{j}=Z \cap S\left(x_{j}\right)$. If Cap canj $_{j}$ denotes capacity with respect to the root $x_{j}$, then

$$
\operatorname{Cap}(Z)=\frac{\sum_{j} \operatorname{Cap}_{x_{j}}\left(Z_{j}\right)}{\left\{1+d_{\pi}(x)\left(\sum_{j} \operatorname{Cap}_{x_{j}}\left(Z_{j}\right)\right)^{p^{\prime}-1}\right\}^{p-1}} .
$$

The theorem and its proof also hold if the set of the children is infinite. We need the obvious lemma: 
Lemma 31. Let $\varphi=\left(I^{*} \sigma\right)^{p^{\prime}-1} \pi^{1-p^{\prime}}$, where $\sigma \in \mathcal{M}_{+}(\bar{T})$ is a measure. For $x$ in $T$, let $C(x)$ be the set of the children of $x$ in $T$. Then,

$$
\pi(x) \varphi(x)^{p-1}=\sigma(x)+\sum_{x_{j} \in C(x)} \pi\left(x_{j}\right) \varphi\left(x_{j}\right)^{p-1} .
$$

We will apply the Lemma for $x$ outside the support of $\sigma$.

Proof of Theorem 30. Let $\varphi$ be the extremal function for $\operatorname{Cap}(Z)$. We claim that

$$
\varphi_{j}=\frac{\varphi}{1-I \varphi(x)}
$$

restricted to $S\left(x_{j}\right)$, is extremal for $\operatorname{Cap}_{x_{j}}\left(Z_{j}\right)$.

For $\zeta \in Z, \sum_{y=x_{j}}^{\zeta} \varphi_{j}(y)=\frac{I \varphi(\zeta)-I \varphi(x)}{1-I \varphi(x)} \geq 1$ q.e. $\zeta$, hence $\varphi_{j}$ is a candidate to be extremal for $\operatorname{Cap}_{x_{j}}\left(Z_{j}\right)$. Suppose there exists another $\psi$ such that $\sum_{y=x_{j}}^{\zeta} \psi(y) \geq 1$ q.e. $\zeta \in Z_{j}$ and $\|\psi\|_{L^{p}\left(S\left(x_{j}\right), \pi\right)}<\left\|\varphi_{j}\right\|_{L^{p}\left(S\left(x_{j}\right), \pi\right)}$. Reasoning as in the proof of Theorem 37 below, we can find a function $\varphi^{\prime}$ such that $\left\|\varphi^{\prime}\right\|_{L^{p}(\pi)}<\|\varphi\|_{L^{p}(\pi)}$ and $I \varphi^{\prime} \geq 1$ q.e. on $Z$, contradicting the extremality of $\varphi$. The claim is proved.

Next, we claim that

$$
\sum_{y=o}^{x} \varphi(y)^{p} \pi(y)=d_{\pi}(x) \operatorname{Cap}(Z)^{p^{\prime}} .
$$

Let $y_{0}=o, \ldots, y_{N}=x$ be an enumeration of the points in the geodesic $[o, x]$ between $o$ and $x$. Since $E$ is contained in $S(x), \varphi$ has is supported on $S(x) \cup[o, x]$.

By Lemma 31,

and, by iteration,

$$
\varphi\left(y_{j}\right)^{p-1} \pi\left(y_{j}\right)=\varphi\left(y_{j-1}\right)^{p-1} \pi\left(y_{j-1}\right)
$$

Summing,

$$
\varphi\left(y_{j}\right)=\frac{\pi(o)^{p^{\prime}-1}}{\pi\left(y_{j}\right)^{p^{\prime}-1}}
$$

$$
\begin{aligned}
\sum_{y=o}^{x} \pi(y) \varphi(y)^{p} & =\sum_{y=o}^{x} \pi(y)^{1+p\left(1-p^{\prime}\right)} \pi(o)^{p\left(p^{\prime}-1\right)} \varphi(o)^{p} \\
& =d_{\pi}(x) \pi(o)^{p^{\prime}} \varphi(o)^{p}=d_{\pi}(x) \operatorname{Cap}(Z)^{p^{\prime}},
\end{aligned}
$$

by Lemma 29. This proves (31). Hence,

$$
\begin{aligned}
\operatorname{Cap}(Z) & =\sum_{j} \sum_{S\left(x_{j}\right)} \varphi^{p} \pi+\sum_{o}^{x} \varphi^{p} \pi \\
& =\sum_{j} \sum_{S\left(x_{j}\right)} \varphi_{j}^{p} \pi[1-I \varphi(x)]^{p}+d_{\pi}(x) \operatorname{Cap}(Z)^{p^{\prime}} \\
& =\sum_{j} \operatorname{Cap}_{x_{j}}\left(Z_{j}\right)\left[1-\operatorname{Cap}(Z)^{p^{\prime}-1} d_{\pi}(x)\right]^{p}+d_{\pi}(x) \operatorname{Cap}(Z)^{p^{\prime}}(\text { by equations }(30) \text { and }(31)),
\end{aligned}
$$

since $I \varphi(x)=\sum_{y=o}^{x} \varphi(y)=\pi(o)^{p^{\prime}-1} \varphi(o) d_{\pi}(x)$. Thus,

$$
\operatorname{Cap}(Z)=\sum_{j} \operatorname{Cap}_{x_{j}}\left(Z_{j}\right)\left[1-\operatorname{Cap}(Z)^{p^{\prime}-1} d_{\pi}(x)\right]^{p}+d_{\pi}(x) \operatorname{Cap}(Z)^{p^{\prime}} .
$$


Rewrite the identity as

$$
\left(\sum_{j} \operatorname{Cap}_{x_{j}}\left(Z_{j}\right)\right)^{p^{\prime}-1}=\operatorname{Cap}(Z)^{p^{\prime}-1}\left[1-\operatorname{Cap}(Z)^{p^{\prime}-1} d_{\pi}(x)\right]^{(1-p)\left(p^{\prime}-1\right)}
$$

and solve with respect to $\operatorname{Cap}(Z)$. The recursive formula in the statement of the theorem is proved.

\subsection{Trace Inequalities on Trees and Ahlfors-regular spaces.}

\subsubsection{Trace Inequalities on Trees. To each positive measure $\mu$ we associate}

$$
[\mu]:=\sup _{a \in T}\left\{\frac{I^{*}\left[\left(I^{*} \mu\right)^{p^{\prime}} \pi^{1-p^{\prime}}\right](a)}{I^{*} \mu(a)}\right\}^{p-1} .
$$

By definition, it is homogeneous of degree $d=1$ with respect to $\mu$ and $d=-1$ with respect to $\pi$. Those measures $\mu$ having $[\mu]<\infty$ are called Carleson measures for $(I, \pi, p)$.

The following theorem was proved, on trees, in [ARS1] and, in a slightly more general form, in [ARS2]. Since the proof in [ARS2] is short, we will give it here, for convenience of the reader. See also [ARS4] for the linear case with special weights: the proof of the special case is the same as that of the general one.

Trace inequalities and, more generally, weighted trace inequalities have a long history. We mention the approach via testing condition of $[\mathrm{KS}]$ and, long before, that by means of capacitary conditions by Maz'ya [Mazya2]. See also [KV] for an approach relating trace inequalities and nonlinear equations.

Theorem 32. There is a constant $C(p)$ which only depends on $p$ such that

$$
\int_{\bar{T}} I f^{p} d \mu \leq C(p)[\mu] \sum_{x \in T} f^{p}(x) \pi(x) .
$$

In fact, we have the quantitative estimates

$$
[\mu] \leq\||| I\|_{\left(L^{p}(\pi), L^{p}(\mu)\right)}^{p} \leq C(p)[\mu] .
$$

We say that a measure is a Carleson measure for the discrete potential $g$ if it satisfies the imbedding (33). Recall that $g(\zeta, \alpha)=\chi_{P(\zeta)}(\alpha)$. We say that $\mu$ satisfies the testing condition if $[\mu]<\infty$. The theorem says that Carleson measures are exactly those satisfying the testing condition.

Proof of the Theorem 32. Inequality (33) means that

$$
I: \ell^{p}(\pi) \rightarrow L^{p}(\mu)
$$

is bounded and, by duality, this is equivalent to the boundedness, with the same norm (which we call $\||\mu\|\mid\|$ in the course of the proof), of

$$
I_{\mu}^{*}: L^{p^{\prime}}(\mu) \rightarrow \ell^{p^{\prime}}\left(\pi^{1-p^{\prime}}\right)
$$

where, it can be easily checked,

$$
I_{\mu}^{*} g(\alpha)=I^{*}(g d \mu):=\int_{S(\alpha)} g d \mu .
$$

For the duality, we use the $\ell^{2}$ inner product to have $\left[\ell^{p}(\pi)\right]^{*}=\ell^{p^{\prime}}\left(\pi^{1-p^{\prime}}\right)$ and the $L^{2}(\mu)$ inner product to have $\left[L^{p}(\mu)\right]^{*}=L^{p^{\prime}}(\mu)$. 
The boundedness of $I_{\mu}^{*}$ is expressed by the inequality

$$
\sum_{\alpha \in T}\left|I^{*}(g d \mu)(\alpha)\right|^{p^{\prime}} \pi^{1-p^{\prime}}(\alpha) \leq\left.\|\| \mu||\right|^{p^{\prime}} \int_{\bar{T}}|g|^{p^{\prime}} d \mu,
$$

with a finite constant \|\|$\mu\|\|$, which obviously has to be checked on positive $g$ 's only. We make the left hand side of (34) larger. Introduce the maximal operator

$$
\mathcal{M}_{\mu} g(\zeta):=\sup _{\beta \in P(\zeta)} \frac{I^{*}(g d \mu)}{I^{*} \mu}
$$

with $\zeta$ in $\bar{T}$ and $g$ positive and measurable on $\bar{T}$.

We will show that, in fact,

$$
\sum_{\alpha \in T}\left[\mathcal{M}_{\mu} g(\alpha)\right]^{p^{\prime}} I^{*}(g d \mu)^{p^{\prime}}(\alpha) \pi^{1-p^{\prime}}(\alpha) \leq\|\| \mu\|\| \int_{\bar{T}} g^{p^{\prime}} d \mu .
$$

In fact, (35) follows from the more general inequality

$$
\int_{\bar{T}}\left[\mathcal{M}_{\mu} g\right]^{p^{\prime}} d \sigma \leq C(p) \int_{\bar{T}} g^{p^{\prime}} \mathcal{M}_{\mu}(d \sigma) d \mu,
$$

which holds for a positive, Borel measure $\sigma$ on $\bar{T}$ with a constant $C(p)$ which only depends on $p$ in $(1, \infty)$. Inequality $(36)$ is proved in Theorem 33 below.

Let us verify that (36) implies (35). Let $\sigma$ be the discrete measure

$$
\sigma(\alpha)=I^{*} \mu(\alpha)^{p^{\prime}} \pi^{1-p^{\prime}}(\alpha) \text {, if } \alpha \in T .
$$

The left hand side of (36) is the left hand side of (35). On the right hand side, observe that

$$
\sup _{\zeta \in \bar{T}} \mathcal{M}_{\mu}(\sigma)(\zeta)=[\mu]
$$

hence,

$$
\int_{\bar{T}} g^{p^{\prime}} \mathcal{M}_{\mu}(d \sigma) d \mu \leq[\mu] \int_{\bar{T}} g^{p^{\prime}} d \mu .
$$

The proof that \|\|$\mu \| \mid \leq C(p)[\mu]$ is complete.

To prove the other direction, it suffices to test inequality (34) on functions of the form $g=\chi_{S(\alpha)}, \alpha$ in $T$.

Observe that testing (35) on the same functions $g=\chi_{S(\alpha)}$, one obtains that the stronger (35) and the weaker (34) hold together with comparable constants, or together fail.

Theorem 33. Given positive Borel measures $\mu$ and $\sigma$ on $\bar{T}$, let

$$
\mathcal{M}_{\mu}(\sigma):=\sup _{\beta \in P(\zeta)} \frac{I^{*}(d \sigma)}{I^{*} \mu} .
$$

Then, (36) holds.

Proof. We show that $\mathcal{M}_{\mu}$ satisfies a weak $(1,1)$ inequality. Let $\lambda>0$ and let $E=\{\zeta \in$ $\left.\bar{T}: \mathcal{M}_{\mu}(g)(\zeta)>\lambda\right\}$. Then, $E$ is disjoint union (by the monotonicity of $\mathcal{M}_{\mu} g$ with respect to to the natural partial order on $T$ ) of sets $S\left(\alpha_{j}\right), j=1, \ldots, n$ in $T$ and

$$
\int_{S\left(\alpha_{j}\right)} g d \mu>\lambda \mu\left(S\left(\alpha_{j}\right)\right)
$$


(by the minimality of $\alpha_{j}$ in $S\left(\alpha_{j}\right)$ ).

Thus, $\mu\left(S\left(\alpha_{j}\right)\right)>0$ and

$$
\begin{aligned}
\sigma(E) & =\sum_{j} \sigma\left(S\left(\alpha_{j}\right)\right) \\
& =\sum_{j} \frac{\sigma\left(S\left(\alpha_{j}\right)\right)}{\mu\left(S\left(\alpha_{j}\right)\right)} \mu\left(S\left(\alpha_{j}\right)\right) \\
& \leq \frac{1}{\lambda} \sum_{j} \mathcal{M} \sigma\left(\alpha_{j}\right) \int_{S\left(\alpha_{j}\right)} g d \mu \\
& \leq \frac{1}{\lambda} \sum_{j} \int_{S\left(\alpha_{j}\right)} g \mathcal{M}_{\mu} \sigma d \mu \\
& \leq \frac{1}{\lambda} \int_{\bar{T}} g \mathcal{M}_{\mu} \sigma d \mu .
\end{aligned}
$$

The $L^{\infty}$ inequality is obvious. Suppose $g \leq C \mathcal{M}_{\mu}(\sigma)-$ a.e.. We can assume that $\sigma \neq 0$ (otherwise (36) holds trivially), hence, $\mathcal{M}_{\mu} \sigma(\zeta)>0$ for all $\zeta$ in $\bar{T}$. This implies that $g \leq C$ $\mu-a . e .$, so that $\int_{S(\alpha)} g d \mu / \mu(S(\alpha)) \leq C$ for all $\alpha$ in $T$ (set it equal to 0 if $\mu(S(\alpha))$ ). Then, $\mathcal{M}_{\mu} g \leq C$ everywhere, hence $\sigma-$ a.e. Marcinkiewicz interpolation gives now the theorem.

5.4.2. Trace Inequalities on Ahlfors-regular spaces. Let $K: X \times X \rightarrow[0, \infty]$ be the kernel defined in (2), with $s \in\left[1 / p^{\prime}, 1\right)$, where $X$ is a Ahlfors $Q$-regular space. We say that a positive Borel measure $\mu$ on $X$ satisfies the trace inequality for the space $(X, m, \rho)$, the exponent $p$ and the kernel $K$ if the inequality

$$
\int_{X}\left(\int_{X} K(x, y) f(y) d m(y)\right)^{p} d \mu \leq C(\mu) \int_{X} f^{p} d m
$$

holds for all positive, Borel $f$.

There is an enormous amount of literature on weighted trace inequalities (see, e.g., [KS], $[\mathrm{KV}],[\mathrm{COV}]$ and the literature quoted there) and we make no claim of originality for the results we are going present below. What we are interested in is the relationship between discrete and continuous trace inequalities, and between different necessary and sufficient conditions.

Let $T$ be the tree associated with $X$ and $K_{T}$ be the kernel on $\partial T$ which corresponds to the same choice of $p$ and $s$.

Theorem 34. The measure $\mu$ satisfies the trace inequality for $K$ if and only if $\Lambda^{*} \mu$ satisfies the trace inequality for $K_{T}$.

Proof. For a Borel measure $\omega$ on $X$, let $K \omega(x)=\int_{X} K(x, y) d \omega(y)$. By duality and symmetry of the kernel $K, \mu$ satisfies the trace inequality for $K$ if and only if the inequality

$$
\int_{X} K(g d \mu)^{p^{\prime}} d m \leq C(\mu) \int_{X} g^{p^{\prime}} d \mu
$$

hold for all positive Borel functions $g$.

That is,

$$
C(\mu) \int_{X} g^{p^{\prime}} d \mu \geq \mathcal{E}_{X}(g d \mu)
$$




$$
\approx \mathcal{E}_{\partial T}\left(\Lambda^{*}(g d \mu)\right)
$$

For $g: X \rightarrow \mathbb{R}^{+}$, define $\Lambda^{*} g:=g \circ \Lambda$. Then one can easily show that

$$
\Lambda^{*}(g d \mu)=\left(\Lambda^{*} g\right) d\left(\Lambda^{*} \mu\right) .
$$

To verify (40), first check the statement for simple $g$, then use Monotone Convergence Theorem. By the (40),

$$
\mathcal{E}_{\partial T}\left(\Lambda^{*}(g d \mu)\right)=\mathcal{E}_{\partial T}\left(\left(\Lambda^{*} g\right)\left(d \Lambda^{*} \mu\right)\right)
$$

and, since

$$
\int_{X} g^{p^{\prime}} d \mu \approx \int_{\partial T} \Lambda^{*}\left(g^{p^{\prime}} d \mu\right)=\int_{\partial T}\left(\Lambda^{*} g\right)^{p^{\prime}} d \Lambda^{*} \mu
$$

the wished inequality (38) is equivalent to the tree inequality

$$
\left.\mathcal{E}_{\partial T}\left(\left(\Lambda^{*} g\right)\left(\Lambda^{*} \mu\right)\right)\right) \leq C(\mu) \int_{\partial T}\left(\Lambda^{*} g\right)^{p^{\prime}} d \Lambda^{*} \mu .
$$

Set $G=\Lambda^{*} g$ and $M=\Lambda^{*} \mu$. The inequality

$$
\mathcal{E}_{\partial T}(G d M) \leq C(M) \int_{\partial T} G^{p^{\prime}} d M
$$

expresses the fact that $M$ satisfies the trace inequality for $K_{\partial T}$. Hence, if $\Lambda^{*} \mu$ satisfies the trace inequality on $\partial T, \mu$ does on $X$.

In the other direction, if (38) holds, then $\mu$ satisfies the capacitary inequality $\mu(E) \leq$ $C(\mu) \operatorname{Cap}_{X}(E)$, when $E$ is a closed subset of $X$ : test the inequality dual to (38) on those $f$ such that $f \geq 1$ on $E$. By the equivalence of continuous and discrete capacities, this implies that $\Lambda^{*} \mu(F) \leq C(\mu) \operatorname{Cap}_{\partial T}(F)$ for closed subsets of $\partial T$. It is well known (see e.g. [KS]) that the capacitary condition implies, via duality, the testing condition $\left[\Lambda^{*} \mu\right]<\infty$ (see also the next subsection) and this, in turn, implies the discrete trace inequality by Theorem 32 .

Remark 35. In view of the results of Section 5.1, the fact that $M$ satisfies the trace inequality for $K_{\partial T}$ is equivalent to the fact that $M$ satisfies (33). Hence, we have a dyadic characterization for the measures satisfying the trace inequalities on Ahlfors-regular spaces.

Corollary 36. A nonnegative Borel measure $\mu$ on $X$ satisfies the trace inequality (37) if and only if it satisfies the testing condition of Kerman-Sawyer type

$$
\int_{B}\left[\int_{B} K(x, y) d \mu(y)\right]^{p^{\prime}} d m(x) \leq C(\mu) \mu(B)
$$

uniformly over the metric balls $B$ in $X$. The constant $C(\mu)$ is comparable with $[\mu]$.

Proof. Clearly, the testing condition (41) is implied by the dual (38) to the trace inequality (37). In the other direction, it is easy to see that the testing condition (41) implies the testing condition on trees, $\left[\Lambda^{*} \mu\right]<\infty$, which implies the discrete trace inequality, which implies in turn, by Theorem 34, the trace inequality in $X$. 


\subsection{Defining Capacities via Carleson measures.}

Theorem 37. Let $E \subseteq \bar{T}$ be compact. Then,

$$
\operatorname{Cap}(E)=\sup _{\operatorname{supp}(\mu) \subseteq E} \frac{\mu(E)}{[\mu]} .
$$

To prove the theorem, we need another characterization of capacity, which holds for the classical capacity, but which is not included in $[\mathrm{AH}]$. Lacking a reference, we give a proof which works in the present context.

\section{Proposition 38.}

$$
\operatorname{Cap}(K)=\inf \{\mathcal{E}(\mu): V(\mu) \geq 1 \text { on } K\} .
$$

Proof. Suppose first that $K \subseteq T$ and that it is finite. Given a measure $\mu$, let $\varphi=$ $\left(I^{*} \mu\right)^{p^{\prime}-1} \pi^{1-p^{\prime}}$. Then, $\mathcal{E}(\mu)=\|\varphi\|_{L^{p}(\pi)}^{p}$ and $V(\mu)=I \varphi$. Let

$$
\mathcal{C}(K)=\inf \{\mathcal{E}(\mu): V(\mu) \geq 1 \text { on } K\}=\mathcal{E}(\bar{\mu}),
$$

where $\bar{\mu}$ is the extremal measure (it exists by elementary compactness and finiteness of $K$ ), which satisfies $V(\bar{\mu})=1$ on $K$ and it is unique.

Let $\bar{\varphi}=\left(I^{*} \bar{\mu}\right)^{p^{\prime}-1} \pi^{1-p^{\prime}}$. Then,

$$
\operatorname{Cap}(K)=\inf \left\{\|\varphi\|_{L^{p}(\pi)}^{p}: I \varphi \geq 1 \text { on } K\right\} \leq \mathcal{C}(K)=\mathcal{E}(\bar{\mu})=\|\bar{\varphi}\|_{L^{p}(\pi)}^{p} .
$$

Suppose there is $\mu_{0}$ such that, with $\varphi_{0}=\left(I^{*} \mu_{0}\right)^{p^{\prime}-1} \pi^{1-p^{\prime}}$,

$$
\operatorname{Cap}(K)=\left\|\varphi_{0}\right\|_{L^{p}(\pi)}^{p}=\mathcal{E}\left(\mu_{0}\right)
$$

and

$$
I \varphi_{0}=V\left(\mu_{0}\right) \geq 1 \text { on } K
$$

Proof of Theorem 37.

We start with inequality $[\leq]$. For fixed $a \in T$ we denote by $\bar{T}_{a}=\bar{S}(a)$ the subtree of $\bar{T}$ having root $a$ and we add a subscript $a$ to the corresponding tree objects: $C_{a p}$ is the capacity of subsets of $\bar{T}_{a}, \mathcal{E}_{a}$ is the energy in $\bar{T}_{a}, \omega_{a}$ is the extremal measure in the definition of capacity, and so on. Let $E_{a}=E \cap \bar{T}_{a}$. The extremal measure $\omega_{a}$ and the function $\varphi_{a}=\left(I_{a}^{*} \omega\right)^{p^{\prime}-1} \pi^{1-p^{\prime}}$ satisfy

$$
\operatorname{Cap}_{a}\left(E_{a}\right)=\omega_{a}\left(E_{a}\right)=\mathcal{E}_{a}\left(\omega_{a}\right)=\left\|\varphi_{a}\right\|_{L^{p}\left(\bar{T}_{a}, \pi\right)}^{p} .
$$

We claim that $\omega_{a}$ is a rescaling of the extremal measure for $E$ in $\bar{T}, \omega$, restricted to $E_{a}$ :

$$
\omega_{a}=\frac{\left.\omega\right|_{E_{a}}}{1-I\left[\left(I^{*} \omega\right)^{p^{\prime}-1} \pi^{1-p^{\prime}}\right]\left(a^{-1}\right)}=\frac{\left.\omega\right|_{E_{a}}}{1-V(\omega)\left(a^{-1}\right)} .
$$

In fact, $\omega_{a}$ minimizes $\mathcal{E}_{a}(\mu)$ over all measures $\mu$ such that $I_{a}\left[\left(I_{a}^{*} \mu\right)^{p^{\prime}-1} \pi^{1-p^{\prime}}\right](\xi)=V_{a}(\mu)(\xi) \geq$ 1 on $E_{a}$ (with the possible exception of a set having null-capacity). On the other hand, we claim that $\left.\omega\right|_{E_{a}}$ minimizes $\mathcal{E}_{a}(\mu)$ among all measures $\mu$ on $E_{a}$ such that $V_{a}(\mu)(\xi) \geq$ $1-V(\omega)\left(a^{-1}\right)$ q.e. on $E_{a}$. 
POTENTIAL THEORY ON TREES, GRAPHS AND AHLFORS-REGULAR METRIC SPACES

Suppose this is not the case. Then there exists a measure $\nu$ on $E_{a}$ such that $V_{a}(\nu)(\xi) \geq$ $1-V(\omega)\left(a^{-1}\right)$ for q.e. $\xi \in E_{a}$ and

$$
\mathcal{E}_{a}(\nu)=\sum_{T_{a}}\left(I^{*} \nu\right)^{p^{\prime}} \pi^{1-p^{\prime}}<\sum_{T_{a}}\left(I^{*} \omega\right)^{p^{\prime}} \pi^{1-p^{\prime}}=\mathcal{E}_{a}\left(\left.\omega\right|_{E_{a}}\right)
$$

Let $\varphi$ be such that $V(\omega)=I \varphi$ in $\bar{T}$ and $\varphi_{1}$ so that $V_{a}(\nu)=I_{a} \nu$ in $\bar{T}_{a}$. Define now a new function $\psi$ on $T$ :

$$
\psi(x)=\left\{\begin{array}{l}
\varphi_{1}(x) \text { if } x \in T_{a} \\
\varphi(x) \text { if } x \in T \backslash T_{a}
\end{array}\right.
$$

We have

$$
I \psi(\xi) \geq 1 \text { q.e. on } E,
$$

hence $\|\psi\|_{L^{p}(\pi)}^{p} \geq \operatorname{Cap}(E)$. On the other hand,

$$
\|\psi\|_{L^{p}(\pi)}^{p}=\mathcal{E}_{a}(\nu)+\left[\mathcal{E}(\omega)-\mathcal{E}_{a}(\omega)\right]<\mathcal{E}(\omega)=\operatorname{Cap}(E),
$$

and we have reached a contradiction.

The measure

$$
\lambda=\frac{\left.\omega\right|_{E_{a}}}{1-V(\omega)\left(a^{-1}\right)},
$$

then, minimizes $\mathcal{E}_{a}(\mu)$ over the set of the measures $\mu$ such that $V_{a}(\mu)(\xi) \geq 1$ for q.e. $\xi$ in $E_{a}$, hence $\lambda=\omega_{a}$. The claim is proved.

By the homogeneity of the energy,

$$
\begin{aligned}
\mathcal{E}_{a}\left(\left.\omega\right|_{E_{a}}\right) & =\left(1-V(\omega)\left(a^{-1}\right)\right)^{p^{\prime}} \mathcal{E}_{a}\left(\omega_{a}\right) \\
& =\left(1-V(\omega)\left(a^{-1}\right)\right)^{p^{\prime}} \omega_{a}\left(E_{a}\right) \\
& =\left(1-V(\omega)\left(a^{-1}\right)\right)^{p^{\prime}-1} \omega\left(E_{a}\right) .
\end{aligned}
$$

As a consequence,

$$
\frac{\sum_{x \geq a}\left(I^{*} \omega\right)^{p^{\prime}} \pi^{1-p^{\prime}}}{I^{*} \omega}=\frac{\mathcal{E}_{a}\left(\left.\omega\right|_{E_{a}}\right)}{\omega\left(E_{a}\right)}=\left(1-V(\omega)\left(a^{-1}\right)\right)^{p^{\prime}-1} \leq 1,
$$

with equality if and only if $a=o$ (we use the default value $V(\omega)\left(o^{-1}\right)=0$ ).

Hence, $[\omega]=1$ and

$$
\operatorname{Cap}(E)=\omega(E)=\frac{\omega(E)}{[\omega]}
$$

We now prove $[\geq]$. By definition of $[\cdot], \mathcal{E}(\mu) \leq[\mu]^{p^{\prime}-1} \mu(E)$ for all measures $\mu$. Then,

$$
\frac{\mu(E)}{[\mu]} \leq \frac{\mu(E)}{\left(\frac{\mathcal{E}(\mu)}{\mu(E)}\right)^{p-1}}=\frac{\mu(E)^{p}}{\mathcal{E}(\mu)^{p-1}} \leq \operatorname{Cap}(E),
$$

as wished.

The proof above has an interesting consequence.

Corollary 39. If $\omega$ is the extremal measure for $C a p(E)$, with $E$ closed in $\bar{T}$, then $[\omega]=1$. 
5.6. Monotonicity of the Tree Condition. First, we give a direct proof that the testing condition for the trace inequalities on trees $([\mu]<\infty)$ is monotone: if $\nu \leq \mu$, then $[\nu] \lesssim[\mu]$.

Then, we use this fact to give a direct proof that the testing condition and the capacitary condition are equivalent. This answers a question which was posed to us by Maz'ya a few years ago (private communication).

It is known (see $[\mathrm{KS}]$ ) that, by a duality argument, the capacitary condition implies the testing condition. We concentrate, then, on the opposite implication. For a measure $\mu$ on $\bar{T}$, let $\sigma_{\mu}=\left(I^{*} \mu\right)^{p^{\prime}} \pi^{1-p^{\prime}}$.

Theorem 40. Let $\mu$ be a measure on $\bar{T}$ and let $\lambda$ be a measurable function on $\bar{T}, 0 \leq \lambda \leq 1$. If $I^{*} \sigma_{\mu} \leq I^{*} \mu$ on $T$, then $I^{*} \sigma_{\lambda \mu} \leq p \cdot I^{*}(\lambda \mu)$.

Corollary 41. If $\nu \leq \mu$ and $\mu, \nu$ are measures on $\bar{T}$, then $[\nu] \leq p^{p-1}[\mu]$.

Proof. By rescaling, it suffices to verify the hypothesis at the root. We use a simple argument based on distribution functions.

Let

$$
\mathcal{M}_{\mu} \lambda(x)=\max _{o \leq y \leq x} \frac{I^{*}(\lambda \mu)(y)}{I^{*} \mu(y)}
$$

be the discrete maximal function we used in [ARS2]. If necessary, we can extend the definition to $x \in \partial T$ in the obvious way. Then,

$$
\begin{aligned}
I^{*} \sigma_{\lambda \mu}(o) & =\sum_{x \in T}\left[\frac{I^{*}(\lambda \mu)(x)}{I^{*} \mu(x)}\right]^{p^{\prime}}\left(I^{*} \mu(x)\right)^{p^{\prime}} \pi^{1-p^{\prime}}(x) \\
& \leq \sum_{x \in T}\left[\mathcal{M}_{\mu} \lambda(x)\right]^{p^{\prime}} \sigma_{\mu}(x) \\
& =2 \int_{0}^{1} t^{p^{\prime}-1} \sigma_{\mu}\left(\zeta \in \bar{T}: \mathcal{M}_{\mu} \lambda(\zeta)>t\right) d t .
\end{aligned}
$$

Now, $\left\{\zeta \in \bar{T}: \mathcal{M}_{\mu} \lambda(\zeta)>t\right\}=\bigsqcup_{j} S\left(x_{j}\right)$ is the disjoint union of Carleson boxes in $\bar{T}$ (by the definition of the maximal function, we do not need to consider the closure of $S\left(x_{j}\right)$ in $\bar{T})$. Then,

$$
\begin{aligned}
t \sigma_{\mu}\left(\zeta \in \bar{T}: \mathcal{M}_{\mu} \lambda(\zeta)>t\right) & =\sum_{j} t \sigma_{\mu}\left(S\left(x_{j}\right)\right) \\
& \leq \sum_{j} t I^{*} \mu\left(x_{j}\right) \\
& \leq \sum_{j} I^{*}(\lambda \mu)\left(x_{j}\right) \\
& \leq I^{*}(\lambda \mu)(o) .
\end{aligned}
$$

Inserting this estimate in the previous one and integrating, we have

$$
I^{*} \sigma_{\lambda \mu}(o) \leq \frac{p^{\prime}}{p^{\prime}-1} \cdot I^{*} \sigma_{\mu}(o)=p I^{*} \sigma_{\mu}(o) .
$$

An immediate consequence of Theorem 40 is that in (42) we do need to restrict to measures supported in $E$. 
POTENTIAL THEORY ON TREES, GRAPHS AND AHLFORS-REGULAR METRIC SPACES

Corollary 42. Let $E \subseteq \bar{T}$ be compact. Then,

$$
\operatorname{Cap}(E) \leq \sup _{\mu} \frac{\mu(E)}{[\mu]} \leq p^{p-1} \operatorname{Cap}(E) .
$$

\subsection{Trace Inequalities: The Testing Condition implies the Capacitary Condition.} We now give a direct proof that the testing condition $[\mu]<\infty$, equivalent to the test inequality (33), is equivalent to a capacitary condition (see (48) below). Both condition s are known, in a fairly general context, to characterize the Trace inequality; hence they are a priori equivalent. Here, however, we give it direct proof of their equivalence. Better, we show that (33) implies (48), since the opposite (direct) implication is known (see [2]).

Theorem 43. Let $\mu$ be a positive, Borel measure on $\bar{T}$. If $\mu$ satisfies

$$
\sup _{x \in T} \frac{I^{*}\left(\left[I^{*} \mu\right]^{p^{\prime}} \pi^{1-p^{\prime}}\right)(x)}{I^{*} \mu(x)} \leq C_{1}(\mu) .
$$

then $\mu$ satisfies, for all closed sets $E$ in $\bar{T}$,

$$
\mu(E) \leq C_{2}(\mu) \operatorname{Cap}(E) .
$$

Moreover, $C_{2}(\mu) \leq p^{p-1} C_{1}(\mu)$. Conversely, (48) implies (47).

Proof. Without loss of generality, suppose that $\mu$ satisfies $(47)$ with $C_{1}(\mu)=1$. Then, $\mu_{E}:=\left.\mu\right|_{E} \leq \mu$ satisfies $\left[\mu_{E}\right] \leq p^{p-1}$ by Theorem 40. Hence,

$$
\begin{aligned}
\operatorname{Cap}(E) & =\sup _{\operatorname{supp}(\nu) \subseteq E} \frac{\nu(E)}{[\nu]} \\
& \geq \frac{\mu(E)}{\left[\mu_{E}\right]} \\
& \geq p^{1-p} \mu(E) .
\end{aligned}
$$

Conditions of testing and of capacitary type are also both known to characterize the Carleson measures for the holomorphic Dirichlet space. Let us recall definitions and results. The Dirichlet space $\mathcal{D}$ contains those functions $f$ which are holomorphic in the unit disc $D$ of the complex plane for which the norm

$$
\|f\|_{\mathcal{D}}^{2}=|f(0)|^{2}+\int_{D}\left|f^{\prime}(z)\right|^{2} d x d y
$$

is finite. A measure $\mu$ on $\bar{D}$ is Carleson for $\mathcal{D}$ if the imbedding $\mathcal{D} \hookrightarrow L^{2}(\mu)$ is bounded. The problem of the boundary values is indeed of interest, when $\mu(\partial D) \neq 0$ : we direct the interested reader to [ARS2] and [1] and to the references therein for a discussion of this problem. We just mention that such problem is intimately related with the characterization itself of the Carleson measures. Carleson measures satisfy a sort of "holomorphic trace inequality", and it is not surpising that they can be characterized by both capacitary and testing conditions. For $z=r e^{i \theta}$ in $D$, let $S(z)=\left\{\rho e^{i \varphi}: r \leq \rho \leq 1,|\theta-\varphi| \leq 2 \pi(1-r)\right\}$ be the usual Carleson box with vertex $z$ and let $I(z)=\partial S(z) \cap \partial D \subset S(z)$ be the part of its boundary lying on $\partial D$. Stegenga [Ste] proved that the Carleson measures for $\mathcal{D}$ are exactly those satisfying

$$
\mu\left(\cup_{j=1}^{n} S\left(z_{j}\right)\right) \leq C(\mu) \operatorname{Cap}\left(\cup_{j=1}^{n} I\left(z_{j}\right)\right),
$$


for all finite subsets $\left\{z_{j}\right\}$ of $D$ such that the $\operatorname{arcs} I\left(z_{j}\right)$ are pairwise disjoint. Here the capacity is nothing other than the logarithmic capacity which corresponds to $s=\frac{1}{2}$ and $p=2$ on the unit circle $\mathbb{T}$. On the other hand, a finite measure $\mu$ is Carleson if and only if it satisfies -for all $a$ in $D$ - the testing condition

$$
\int_{S(a)}[\mu(S(z) \cap S(a))]^{2} \frac{d x d y}{\left(1-|z|^{2}\right)^{2}} \leq C(\mu) \mu(S(a)) .
$$

This condition can be written in a discrete fashion (see, e.g., [ARS1] and [ARS2]), in the spirit of the present paper. We remind the reader that the first testing condition for Carleson measures was found in [KS] and it is different from (50) (it is what we get from (50) after applying the Muckenhoupt-Wheeden-Wolff inequality. The left hand side of (50) is analogous to the left hand side of (11), while the left hand side of the testing condition in [KS] is analogous to the right hand side of (11)).

Now, Stegenga's condition involves a measure in the interior and a capacity for a boundary set. In Theorem 43, by contrast, we have that measure and capacity either both live in the interior, or both live on the boundary. It is then interesting, we believe, to know whether the capacity of the interior set is comparable with that of its "shadow" on the boundary. The answer on trees, which might be transfered to various metric situations, is positive, under a mild assumption on the weight $\pi$.

Lemma 44. Suppose that, for all $x$ in $T$,

$$
\operatorname{Cap}(\partial S(x)) \gtrsim d_{\pi}(x)^{1-p}
$$

(i.e., that $\left.d_{\pi}(x)^{1-p} \approx \operatorname{Cap}(\{x\}) \approx \operatorname{Cap}(\partial S(x))\right)$. Then,

$$
\operatorname{Cap}(S(E)) \lesssim \operatorname{Cap}(E)
$$

(i.e., $\operatorname{Cap}(S(E)) \lesssim \operatorname{Cap}(E))$ whenever $E=\cup_{j} \partial S\left(x_{j}\right)$ is finite union of sets of the form $\partial S\left(x_{j}\right)$.

Observe that the converse inequality, $\operatorname{Cap}(S(E)) \geq \operatorname{Cap}(E)$, follows from trivial comparison.

Proof. Let $\varphi$ be the extremal function for $E$ and let $A=\left\{x_{j}: I \varphi\left(x_{j}^{-1}\right) \geq 1 / 2\right\}$ and $B=\left\{x_{j}: I \varphi\left(x_{j}^{-1}\right)<1 / 2\right\}$. Then,

$$
\begin{aligned}
\operatorname{Cap}(E) & =\sum_{x \in T} \varphi^{p}(x) \pi(x) \\
& \geq \sum_{x \in \cup_{a \in A}[o, a]} \varphi^{p}(x) \pi(x)+\sum_{b \in B} \sum_{x \in S(b)} \varphi^{p}(x) \pi(x) \\
& \geq 2^{-p} \operatorname{Cap}(A)+\sum_{b \in B}\left(1-I \varphi\left(b^{-1}\right)\right)^{p} \sum_{x \in S(b)}\left(\frac{\varphi(x)}{1-I \varphi\left(b^{-1}\right)}\right)^{p} \pi(x) .
\end{aligned}
$$

Since, when $\zeta$ belongs to $S(b)$ for some $b \in B$,

$$
\sum_{x \in[b, \zeta]} \frac{\varphi(x)}{1-I \varphi\left(b^{-1}\right)} \geq 1,
$$

the function $\varphi(x) /\left(1-I \varphi\left(b^{-1}\right)\right)$ is admissible for $\operatorname{Cap}_{b}(\partial S(b))$, hence, 


$$
\begin{aligned}
\sum_{x \in S(b)}\left(\frac{\varphi(x)}{1-I \varphi\left(b^{-1}\right)}\right)^{p} \pi(x) & \geq \operatorname{Cap}_{b}(\partial S(b)) \\
& \geq \operatorname{Cap}(\partial S(b)) \\
& \gtrsim d_{\pi}(b)^{1-p},
\end{aligned}
$$

by (51). Inserting this last inequality in (52),

$$
\begin{aligned}
\operatorname{Cap}(E) & \gtrsim\left[\operatorname{Cap}(A)+\sum_{b \in B} d_{\pi}(b)^{1-p}\right] \\
& \gtrsim \operatorname{Cap}(A)+\sum_{b \in B} \operatorname{Cap}(\{b\}) \\
& \geq \operatorname{Cap}(A \cup B)=\operatorname{Cap}(S(E)) .
\end{aligned}
$$

Corollary 45. Suppose that condition (51) holds for the weight $\pi$. Then, for a mesaure $\mu$ on $\bar{T}$, the testing condition (47) is equivalent to the capacitary condition

$$
\mu(S(E)) \lesssim \operatorname{Cap}(E),
$$

to be checked over all $E$ subsets of $\partial T$ having the form $E=\cup_{j} \partial S\left(x_{j}\right)$ (with the set of the $x_{j}$ 's being finite).

\section{Appendix: Potential Theory on Trees}

In $[\mathrm{AH}]$, Sect. 2.3-2.5, the basics of Nonlinear Potential Theory are developed, based on a kernel $g: X \times M \rightarrow[0,+\infty]$, where:

- $(M, \nu)$ is a measure space;

- $(X, \delta)$ is a separable, complete, locally compact metric space;

- $g(\cdot, y)$ is lower semicontinuous on $X$ for each $y \in M$;

- $g(x, \cdot)$ is measurable for each $x \in X$.

Moreover, an exponent $p$ is chosen in $(1, \infty)$.

With these data, the capacity of a set $E$ in $X$ is defined as

$$
\operatorname{Cap}(E):=\left\{\|f\|_{L^{p}(\nu)}^{p}: f \geq 0, \int_{M} g(x, y) f(y) d \nu(y) \geq 1 \text { if } x \in E\right\} .
$$

Basic object in the theory are the potentials of a nonnegative, measurable function:

$$
\mathcal{G} f(x)=\int_{M} g(x, y) f(y) d \nu(y), x \in X
$$

and of a Borel, nonnegative measure:

$$
\check{\mathcal{G}} m(y)=\int_{X} g(x, y) d m(x), y \in M .
$$

On the tree $T$ having root $o$, we set $X=\bar{T}$, where $\bar{T}$ is endowed with any reasonable metric (e.g. the length metric obtained by assigning to each edge $\left(\alpha, \alpha^{-1}\right)$ the length $2^{-d(\alpha)}$ ) and $M=T$ with the discrete measure $\nu=\pi^{1-p^{\prime}}$. Our kernel is

$$
g(\xi, x)=\chi_{P(\xi)}(x)=\chi_{S(x)}(\xi) .
$$


We shall also let $\varphi=f \nu$, hence, $\varphi^{p} \pi=f^{p} \nu$.

With these definitions and renormalizations, the following property summarizes the main results from $[\mathrm{AH}]$, Sect. 2.3-2.5.

\section{Proposition 46.}

(1) $G f(\zeta)=I(f \nu)(\zeta)=I \varphi$;

(2) $\check{G} m(y)=I^{*} m(y)=\int_{S(y)} d m$;

(3) $\operatorname{Cap}(E)=\inf \left\{\sum \varphi^{p} \pi: \varphi \geq 0, I \varphi \geq 1\right.$ on $\left.E\right\}$;

(4) Let $E \subseteq \bar{T}$. Then, $\operatorname{Cap}(E)=0$ if and only if there is $\varphi \in L_{+}^{p}(\pi)$ such that $E \subseteq\{x$ : $I \varphi(x)=+\infty\}$. In particular, $E \subseteq \partial T$;

(5) Let $\bar{\Omega}_{E}$ be the closure of $\Omega_{E}$ in $L^{p}(\pi)$. Then,

$$
\bar{\Omega}(E):=\left\{\varphi \in L_{+}^{p}(\pi): I \varphi(\xi) \geq 1 \text { for q.e. } \xi \in E\right\}
$$

(6) Let $E \subset \bar{T}$. Then, $\exists$ ! $\varphi^{E}$ such that $\varphi^{E} \in L_{+}^{p}(\pi), I \varphi^{E} \geq 1$ q.e. on $E, \operatorname{Cap}(E)=$ $\sum \varphi^{E}(x)^{p} \pi(x)$

(7) The mutual energy of $m$ and $f$ is $\tilde{\mathcal{E}}(m, f)=\int_{\bar{T}} I(f \nu) d m=\sum_{T} I^{*} m \cdot f \cdot \nu=\mathcal{E}(m, \varphi)$;

(8) Let $K \subset \bar{T}$ be compact. Then,

$$
\operatorname{Cap}(K)^{1 / p}=\sup \left\{m(K): \text { supported in } K, \sum_{T}\left[I^{*} m\right]^{p^{\prime}} \pi^{1-p^{\prime}} \leq 1\right\} ;
$$

(9) There exists an extremal, positive measure $m^{K}$ with support in $K$, such that

$$
\varphi^{K}=\left[I^{*} m^{K}\right]^{p^{\prime}-1} \pi^{1-p^{\prime}} .
$$

Moreover,

$$
\operatorname{Cap}(K)=m^{K}(K)=\sum_{T}\left[I^{*} m^{K}\right]^{p^{\prime}} \pi^{1-p^{\prime}}=\int_{\bar{T}} I\left[\varphi^{K}\right] d m^{K}
$$

(10) The nonlinear potential of a measure $m$ is

$$
V(m)(\zeta)=I\left(\pi^{1-p^{\prime}}\left[I^{*} m\right]^{p^{\prime}-1}\right)(\zeta) .
$$

The energy of a measure is

$$
\mathcal{E}(m)=\int_{\bar{T}} V(m) d m=\sum_{x}\left(I^{*} m\right)^{p^{\prime}} \pi^{1-p^{\prime}}=\int_{\bar{T}} I\left[\left(I^{*} m\right)^{p^{\prime}-1} \pi^{1-p^{\prime}}\right] d m ;
$$

(11) Let $K$ be compact in $\bar{T}$. Then,

$$
I \varphi^{K}=V\left(m^{K}\right) \leq 1 \text { on } \operatorname{supp}\left(m^{K}\right)
$$

and

$\operatorname{Cap}(K)=\max \{m(K): m \geq 0$ and supported in $K$, such that $V(m) \leq 1$ on $\operatorname{supp}(m)\}$;

(12) Let $E \subset \bar{T}$ is such that $\operatorname{Cap}(E)<\infty$. Then, there is a measure $m^{E}$ such that $\varphi^{E}$, the capacitary function, is given by $\varphi^{E}=\left(I^{*} m^{E}\right)^{p^{\prime}-1} \pi^{1-p^{\prime}}$. Moreover,

$$
I \varphi^{E} \geq 1 \text { q.e. on } E, I \varphi^{E} \leq 1 \text { q.e. on } \operatorname{supp}\left(m^{E}\right)
$$

and

$$
m^{E}(\bar{E})=\sum_{T}\left(I^{*} m^{E}\right)^{p^{\prime}} \pi^{1-p^{\prime}}=\int_{\bar{T}} I \varphi^{E} d m^{E}=\operatorname{Cap}(E) .
$$


The capacity functional has the following basic properties:

\section{Proposition 47.}

(1) $\operatorname{Cap}(\emptyset)=0$;

(2) If $E \subseteq F$, then $\operatorname{Cap}(E) \leq \operatorname{Cap}(F)$;

(3) If $K_{i} \searrow K$ are compact sets, then $\operatorname{Cap}\left(K_{i}\right) \searrow \operatorname{Cap}(K)$;

(4) If $E_{i} \nearrow E$ are arbitrary sets, then $\operatorname{Cap}\left(E_{i}\right) \nearrow C a p(E)$.

$A$ set $E$ in $\bar{T}$ is capacitable if

$$
\operatorname{Cap}(E)=\sup \{\operatorname{Cap}(K): K \subseteq E \text { is compact }\} .
$$

The Capacitability Theorem guarantees that all Suslin sets (hence, all Borel sets) are capacitable.

As a consequence of the above and of homogeneity, we have the following equivalent definitions of capacity.

$$
\begin{aligned}
& \operatorname{Cap}(K)=\inf \left\{\|\varphi\|_{L^{p}(\pi)}^{p}: I \varphi \geq 1 \text { on } K\right\} \\
& =\inf \left\{\|\varphi\|_{L^{p}(\pi)}^{p}: I \varphi \geq 1 \text { q.e. on } K\right\} \\
& =\inf \left\{\frac{\|\varphi\|_{L^{p}(\pi)}^{p}}{\left(\inf _{x \in K} I \varphi\right)^{p}}: I \varphi \geq 1 \text { q.e. on } K\right\} \\
& =(\sup \{m(K): \mathcal{E}(m) \leq 1, \operatorname{supp}(m) \subseteq K\})^{p} \\
& =\sup _{\operatorname{supp}(m) \subseteq K} \frac{m(K)^{p}}{\mathcal{E}(m)^{p-1}} \\
& =(\inf \{\mathcal{E}(m): m(K) \geq 1, \operatorname{supp}(m) \subseteq K\})^{1-p} \\
& =\sup \{m(K): V(m) \leq 1 \text { on } \operatorname{supp}(m), \operatorname{supp}(m) \subseteq K\} \\
& =\sup _{m: \operatorname{supp}(m) \subseteq K} \frac{m(K)}{\sup _{\xi \in \operatorname{supp}(m)} V(m)(\xi)^{p-1}} \\
& =\left(\inf _{m: \operatorname{supp}(m) \subseteq K}\left\{\sup _{\xi \in \operatorname{supp}(m)} V(m)(\xi)^{p-1}: m(K) \geq 1\right\}\right)^{-1}
\end{aligned}
$$

\section{REFERENCES}

[AH] D. R. Adams, L. I. Hedberg, Function Spaces and Potential Theory, Grundlehren der Mathematischen Wissenschaften 314, Springer-Verlag, Berlin, 1996, xii+366 pp.. 2, 4, 6, 23, 26, 27, 36, 41, 42

[AiE] H. Aikawa, M. Essén, Potential Theory-Selected topics, Lecture Notes in Mathematics 1633, SpringerVerlag, Berlin, 1996.

[A] N. Arcozzi, Capacity of Shrinking Condensers in the Plane, J. Funct. Anal. 263 (2012), no. 10, 31023116. . 3

[AB] N. Arcozzi, M. Bersani, Capacity of Shrinking Condensers in Euclidean space, in preparation. 3

[AR] N. Arcozzi, R. Rochberg, Topics in Dyadic Dirichlet Spaces, New York J. Math. 10 (2004), 45-67. 20

[ARS1] N. Arcozzi, R. Rochberg, E. Sawyer, Carleson Measures for Analytic Besov Spaces, Rev. Mat. Iberoamericana 18 (2002), no. 2, 443-510. 2, 32, 40

[ARS2] N. Arcozzi, R. Rochberg, E. Sawyer, The Characterization of The Carleson Measures for Analytic Besov Spaces: A Simple Proof, Complex and Harmonic Analysis, 167-177, DEStech Publ., Inc., Lancaster, PA, 2007. 32, 38, 39, 40 
[ARS3] N. Arcozzi, R. Rochberg, E. Sawyer, Capacity, Carleson Measures, Boundary Convergence and Exceptional Sets, "Perspective in Harmonic Analysis and Applications" in honor of V.G. Maz'ya 70-th birthday, Proceedings of Symposia in Pure and Applied Mathematics, Editors: Dorina Mitrea, Marius Mitrea. 2

[ARS4] N. Arcozzi, R. Rochberg, E. Sawyer, Carleson Measures for the Drury-Arveson Hardy Space and other Besov-Sobolev Spaces on Complex Balls, Adv. Math. 218 (2008), no. 4, 1107-1180. 32

[ARSW] N. Arcozzi, R. Rochberg, E. Sawyer, B. D. Wick, Bilinear forms on the Dirichlet Space, Anal. and PDE 3 (2010), no. 1, 21-47. 3

[BP] I. Benjamini, Y. Peres, Random Walks on a Tree and Capacity in the interval, Ann. Inst. H. Poincaré Probab. Statist. 28 (1992), no. 4, 557-592. 2, 3, 30

[1] A. Beurling, Ensembles exceptionnels, Acta Math. 72, (1940). 1-13. 39

[BLU] A. Bonfiglioli, E. Lanconelli, F. Uguzzoni, Stratified Lie groups and Potential Theory for their subLaplacians, Springer Monographs in Mathematics. Springer, Berlin, 2007. xxvi+800 pp.. 6

[BoPa] M. Bourdon, H. Pajot, Cohomologie lp et espaces de Besov, J. Reine Angew. Math. 558 (2003), 85108. 3

[Christ] M. Christ, A T(b) theorem with Remarks on Analytic Capacity and the Cauchy Integral, Colloq. Math. 60/61 (1990), no. 2, 601-628. 3, 4, 7

[COV] C. Cascante, J. M. Ortega, I. E. Verbitsky, Nonlinear Potentials and Two Weight Trace Inequalities for General Dyadic and Radial Kernels, Indiana Univ. Math. J. 53 (2004), no. 3, 845-882. 28, 34

[DB] F. Di Biase, Fatou type theorems, Maximal functions and approach regions Progress in Mathematics, 147.

[Doob] J. L. Doob, Classical Potential Theory and its Probabilistic Counterpart, Grundlehren der Mathematischen Wissenschaften 262, Springer-Verlag, New York, 1984. xxiv+846 pp. Birkhäuser Boston, Inc., Boston, MA, 1998. xii+152 pp.. 2

[Geo] A. Georgakopoulos, Graph topologies induced by edge lengths, Discrete Math. 311 (2011), no. 15, 1523-1542. 10, 12

[Gro] M. Gromov, Metric structures for Riemannian and non-Riemannian spaces, Based on the 1981 French original, with appendices by M. Katz, P. Pansu and S. Semmes, translated from the French by Sean Michael Bates, Progress in Mathematics 152, Birkhauser Boston, Inc., Boston, MA, 1999, xx+585 pp.. 7

[HaK] P. Hajlasz, P. Koskela, Sobolev met Poincare, Mem. Amer. Math. Soc. 145 (2000), no. 688, x+101 pp.. 4

[HW] L. I. Hedberg, T. H. Wolff, Thin Sets in Nonlinear Potential Theory, Ann. Inst. Fourier (Grenoble) 33 (1983), no. 4, 161-187. 3, 20, 28

[He] J. Heinonen, Lectures on Analysis on Metric Spaces, Universitext, Springer-Verlag, New York, 2001. $\mathrm{x}+140$ pp.. 2, 4, 6

[HeK] J. Heinonen, P. Koskela, Quasiconformal Maps in Metric Spaces with Controlled Geometry, Acta Math. 181 (1998), no. 1, 1-61. 4, 13

[HS] I. Holopainen, P. Soardi, p-Harmonic Functions on Graphs and Manifolds, Manuscripta Math. 94 (1997), no. 1, 95-110.

[KV] N. J. Kalton, I. E. Verbitsky, Nonlinear Equations and Weighted Norm Inequalities, Trans. Amer. Math. Soc. 351 (1999), no. 9, 3441-3497. 3, 4, 28, 32, 34

[2] R. Kerman, E. Sawyer, The trace inequality and eigenvalue estimates for Schrödinger operators, Ann. Inst. Fourier (Grenoble) 36 (1986), no. 4, 207-228. 39

[KS] R. Kerman, E. Sawyer, Carleson Measures and Multipliers of Dirichlet-type Spaces, Trans. Amer. Math. Soc. 309 (1988), no. 1, 87-98. 26, 32, 34, 35, 38, 40

[LyPe] R. Lyons, Y. Peres, Probability on Trees and Networks, preprint available at http://mypage.iu.edu/ rdlyons/prbtree/prbtree.html 2, 26

[Mazya] V. G. Maz'ja, Sobolev Spaces, translated from the Russian by T. O. Shaposhnikova. Springer Series in Soviet Mathematics. Springer-Verlag, Berlin, 1985. xix+486 pp.. 2

[Mazya2] V. G. Maz'ja, Imbedding Theorems and their Applications, Baku Sympos. (1966) "Nakua", Moscow, 1970, 142-159 (Russian). 32

[MW] B. Muckenhoupt, R. Wheeden, Weighted Norm Inequalities for Fractional Integrals, Trans. Amer. Math. Soc. 192 (1974), 261-274. 3, 20 
[Ru] W. Rudin, Principles of mathematical analysis, Third edition. International Series in Pure and Applied Mathematics. McGraw-Hill Book Co., New York-Auckland-Dsseldorf, 1976. x+342 pp. 10

[So] M. Paolo, P. M. Soardi, Potential Theory on Infinite Networks, Lecture Notes in Mathematics 1590, Springer-Verlag, Berlin, 1994. viii+187 pp.. 2, 26, 27, 30

[Ste] D. A. Stegenga, Multipliers of the Dirichlet Space, Illinois J. Math. 24 (1980), no. 1, 113-139. 2, 39

[Ts] M. Tsuji, Potential Theory in Modern Function Theory, Maruzen Co., Ltd., Tokyo 1959, 590 pp.. 2

[VW] I. E. Verbitsky, R. Wheeden, Weighted Norm Inequalities for Integral Operators, Trans. Amer. Math. Soc. 350 (1998), no. 8, 3371-3391. 2, 3

Dipartimento di Matematica, Universita di Bologna, 40127 Bologna, ITALY

E-mail address: arcozzi@dm.unibo.it

Department of Mathematics, Washington University, St. Louis, MO 63130, U.S.A

E-mail address: rr@math.wustl.edu

Department of Mathematics \& Statistics, McMaster University; Hamilton, Ontairo, L8S 4K1, CANADA

E-mail address: sawyer@mcmaster.ca

School of Mathematics, Georgia Institute of Technology, 686 Cherry Street, Atlanta, GA USA 30332-0160

E-mail address: wick@math.gatech.edu 